\title{
Stabilized numerical solutions of three-phase porous media flow using a multiscale finite element formulation
}

\author{
Ruben Juanes ${ }^{1}$ \\ Tadeusz W. Patzek ${ }^{1,2}$ \\ ${ }^{1}$ Department of Civil and Environmental Engineering \\ University of California at Berkeley \\ 631 Davis Hall \#1710, Berkeley, CA 94720 \\ ${ }^{2}$ Earth Sciences Division \\ Lawrence Berkeley National Lab \\ 1 Cyclotron Road Mailstop 90-1116, Berkeley, CA 94720 \\ To be submitted to \\ Computer Methods in Applied Mechanics and Engineering \\ January 25, 2003
}

\begin{abstract}
We present a multiscale formulation for the numerical solution of one-dimensional three-phase flow through porous media. In the case of vanishing capillarity effects, the system of equations describing three-phase flow becomes almost hyperbolic, and the solution develops shocks and boundary layers. Under these conditions, classical numerical methods produce a solution that is either unstable or excessively diffusive.
\end{abstract}


The key idea of the proposed formulation, originally presented by Hughes [Comput. Methods Appl. Mech. Engrg., 127:387-401 (1995)], is a multiple-scale decomposition into resolved - grid - scales and unresolved - subgrid - scales. Incorporating the effect of the subgrid scales onto the coarse scale problem results in a finite element method with enhanced stability properties, capable of accurately representing the sharp features of the solution. In the formulation developed herein, the multiscale split is invoked prior to any linearization of the equations. Special attention is given to the choice of the matrix of stabilizing coefficients, and a novel discontinuity-capturing technique is proposed and compared with existing formulations.

The methodology is applied to the simulation of two problems of great practical interest: oil filtration in the vadose zone, and watergas injection in a hydrocarbon reservoir. These numerical simulations clearly show the potential and applicability of the formulation for solving the highly nonlinear, nearly hyperbolic system of threephase porous media flow on very coarse grids.

KEY WORDS: porous media, three-phase flow, finite elements, stabilized solutions, multiscale methods, shock-capturing

\section{Introduction}

Flow of three immiscible fluids - denoted hereafter as water, oil, and gasoccurs in a variety of flow situations in the subsurface, including gas injection into hydrocarbon reservoirs, water flooding in the presence of free gas, and migration of nonaqueous phase liquids in the shallow subsurface. As a result, mathematical and numerical modeling of three-phase flow in porous media has become essential to perform quantitative evaluations and predictions of enhanced oil recovery processes, and environmental remediation of the vadose zone.

The development of physically-based and tractable mathematical models of multiphase flow is a very challenging task. One of the main difficulties is the inherent multiscale character, reflected not only on the scale dependency of key parameters, such as the absolute permeability, but also on the fact that different physical processes dominate at different scales: capillary forces govern the microscale, whereas viscous and gravity forces dominate 
the macroscale. The direct consequence of this complex behavior has been the development of mathematical models which are extensions of successful models for single-phase flow. In this paper we use a classical mathematical formulation $[3,7,57]$, which is based on: (1) a multiphase form of Darcy's equation; (2) the fractional flow approach. The mathematical problem of three-phase flow is then written as a pressure equation of elliptic type, and a system of saturation equations of parabolic type. Because the pressure equation is trivial in the one-dimensional case, we concentrate on the numerical solution of the system of saturation equations.

Even though the equations studied in this paper are a simplified version of the full nonisothermal, multiphase, compositional model in several dimensions, they display some of the essential features that pose numerical difficulties. In particular, the problem is extremely nonlinear, almost hyperbolic for the case of interest — vanishing capillarity-, and the solution naturally develops shocks and boundary layers.

It is well known that, for the type of problems described above, classical numerical methods either lack stability — producing globally oscillatory solutions - or accuracy - solutions are overly diffusive-. In an attempt to obtain stable solutions which retain high-order accuracy, the equations are solved here using a stabilized finite element method (see, e.g., [17] and the references therein). Recently, stabilized finite element methods have been reinterpreted from the point of view of multiscale phenomena [26], where the stabilizing terms arise naturally in a variational multiscale method [27]. This idea of a multiple-scale decomposition of the solution, which is now dominant in fluid mechanics, is adopted here for the simulation of multiphase, porous media flow. The major benefit of this numerical formulation is that the oscillatory behavior of the classical Galerkin method is drastically reduced. This is achieved without compromising the computational cost of the method, or the accuracy of the solution. The specific contributions of this paper may be succinctly summarized as follows:

1. The multiscale formalism is applied to the equations governing onedimensional three-phase flow through porous media. Previous work on miscible and immisicible two-phase flow [41,42] — described by scalar equations - is extended here to nonlinear systems of conservation laws.

2. Nonlinearity of the equations is retained at the time of invoking the multiscale split. Proper linearization of the stabilizing terms is introduced after the multiple-scale decomposition into resolved and unre- 
solved scales. Furthermore, the multiple-scale solution is not reconstructed from point values of coarse and subgrid scales.

3. Several definitions of the key parameter of the formulation - the matrix of stabilizing coefficients - are tested and compared [11, 29, 59]. To further reduce or completely eliminate localized oscillations that may persist in the stabilized solution, several existing shock-capturing techniques are studied $[9,18,30,31,59]$, and a novel expression for the discontinuity-capturing diffusion is proposed.

It is important to realize that the formulation presented here is very different from other methods that account for multiple-scale phenomena, such as the multiscale finite element method [25], the subgrid upscaling technique [1,2], and the mortar upscaling method [58], where the main objective is to incorporate the small-scale heterogeneity. On the other hand, a recent paper [53] applies the original variational multiscale formulation of $[26,27]$ to porous media flows. It is restricted, however, to the linear scalar equation describing steady-state, single-phase, Darcy flow, and the objective is to remove velocity-pressure instabilities, rather than instabilities arising from the nearly hyperbolic character of the equations.

An outline of the paper is as follows. In Section 2 we derive the governing equations of three-phase flow, which are then written - in dimensionless form - as a nonlinear system of conservation laws. After a summary of the weak form of the problem and the associated standard Galerkin method, we describe in Section 3 the multiple-scale approach. Special attention is given to the matrix of stabilizing coefficients and to alternative shock-capturing techniques. In Section 4 we present several representative numerical simulations of three-phase flows. The first application is an oil filtration problem in a relatively dry medium, and the second reproduces water-gas injection in a hydrocarbon reservoir. Numerical solutions are compared with a general, newly developed, analytical solution [40]. These simulations illustrate the outstanding performance of the proposed methodology. In Section 5 we gather the main conclusions, and anticipate ongoing and future research.

\section{Mathematical formulation}

The macroscopic equations governing immiscible multiphase flow in porous media are mass conservation equations and constitutive relations that de- 
scribe the flux of each phase. Classical formulations rely on a multiphase flow extension of Darcy's equation [54]. We shall make use of this extension - which is not rigorously derived from first principles [22] — as a modeling assumption, rather than a physical law. By invoking the fractional flow formalism [7], the problem may be written in terms of a pressure equation and a system of saturation equations. The solution to the pressure equation is trivial in the one-dimensional case and, therefore, we shall focus on the numerical solution of the system of saturation equations.

\subsection{Continuum equations}

Here we present a standard derivation of the equations of three-phase flow in porous media under the following assumptions:

1. One-dimensional flow,

2. Zero distributed sources and sinks,

3. Immiscible fluids,

4. Incompressible fluids,

5. Rigid homogeneous porous medium,

6. Multiphase flow extension of Darcy's equation,

7. Negligible gravity effects.

Similar derivations of the governing equations may be found in $[3,7,21,47,57]$, among others.

The equation of mass conservation of a chemical component $i$ in a onedimensional medium without distributed sources (assumptions 1 and 2) is:

$$
\partial_{t} m_{i}+\partial_{x} F_{i}=0, \quad 0<x<L, \quad t>0
$$

where $m_{i}$ is the mass of the $i$-component per unit bulk volume, $F_{i}$ is the mass flux of component $i, \partial_{t}$ and $\partial_{x}$ denote the partial derivatives with respect to time and space, respectively, and $L$ is the length of the domain. By virtue of assumption 3, one can identify any chemical component $i$ with the generic 
fluid phase $\alpha$. We shall consider three fluid phases: water $(w)$, oil $(o)$ and gas $(g)$. The mass of the $\alpha$-phase per unit bulk volume is:

$$
m_{\alpha}=\rho_{\alpha} S_{\alpha} \phi
$$

where $\rho_{\alpha}$ is the density of the $\alpha$-phase, $S_{\alpha}$ is the saturation of the $\alpha$-phase, and $\phi$ is the porosity. In view of assumptions 4 and 5, the phase densities and the porosity are taken as constants. We write the mass flux of the $\alpha$-phase in the form:

$$
F_{\alpha}=\rho_{\alpha} v_{\alpha} \phi
$$

where $v_{\alpha}$ is the velocity of the $\alpha$-phase. Using Muskat's [54] extension of Darcy's equation to model the fluid velocities (assumption 6) with negligible gravity effects (assumption 7):

$$
v_{\alpha}=-\frac{k}{\phi} \frac{k_{r \alpha}}{\mu_{\alpha}} \partial_{x} p_{\alpha},
$$

where $k$ is the absolute permeability, $k_{r \alpha}$ and $\mu_{\alpha}$ are the relative permeability and the dynamic viscosity of the $\alpha$-phase, respectively, and $p_{\alpha}$ is the pressure of the $\alpha$-phase. For convenience, we define the relative mobility of the $\alpha$ phase,

$$
\lambda_{\alpha}:=\frac{k_{r \alpha}}{\mu_{\alpha}} .
$$

We define the capillary pressures $P_{c \alpha}$ in a standard way, as differences of the phase pressures $p_{\alpha}$ and a reference pressure $p$. For the purpose of this derivation, we take the oil pressure as the reference pressure,

$$
p:=p_{o}
$$

and define:

$$
\begin{aligned}
P_{c w} & :=p_{w}-p, \\
P_{c g} & :=p_{g}-p .
\end{aligned}
$$

We note in passing that the water capillary pressure is usually defined with opposite sign. Definition (7) has been used here to preserve symmetry of the formulation.

Substituting Equations (2) and (3) into Equation (1), the mass conservation equations for the $\alpha$-phase reads:

$$
\partial_{t} S_{\alpha}+\partial_{x} v_{\alpha}=0, \quad \alpha=w, o, g .
$$


Equations (9) are subject to the additional constraint that the fluids fill up the entire pore space:

$$
\sum_{\alpha=w, o, g} S_{\alpha} \equiv 1
$$

The essence of the fractional flow approach (see, e.g., $[3,7,57])$ is to combine Equations (9) with the algebraic constraint (10) to obtain a "pressure equation" of elliptic type, and a system of "saturation equations" of parabolic type. Summation of the mass conservation equations and use of the saturation constraint yields the pressure equation:

$$
\partial_{x} v_{T}=0
$$

where we have defined the total velocity $v_{T}$ as

$$
v_{T}:=\sum_{\alpha=w, o, g} v_{\alpha}
$$

Substituting the expression of the fluid velocities (4) and the capillary pressures (7)-(8) in Equation (12):

$$
v_{T}=-\frac{k}{\phi}\left(\lambda_{T} \partial_{x} p+\lambda_{w} \partial_{x} P_{c w}+\lambda_{g} \partial_{x} P_{c g}\right)
$$

or, rearranging,

$$
-\frac{k}{\phi} \lambda_{T} \partial_{x} p=v_{T}+\frac{k}{\phi} \lambda_{w} \partial_{x} P_{c w}+\frac{k}{\phi} \lambda_{g} \partial_{x} P_{c g}
$$

where

$$
\lambda_{T}:=\sum_{\alpha=w, o, g} \lambda_{\alpha}
$$

is the total mobility. Using Equations (4), (11) and (14) in Equations (9), and defining the fractional flow of the $\alpha$-phase,

$$
f_{\alpha}:=\frac{\lambda_{\alpha}}{\lambda_{T}}
$$

we obtain the system of saturation equations:

$$
\begin{aligned}
& \partial_{t} S_{w}+v_{T} \partial_{x} f_{w}-\partial_{x}\left[\frac{k}{\phi}\left(\lambda_{w}\left(1-f_{w}\right) \partial_{x} P_{c w}-\lambda_{w} f_{g} \partial_{x} P_{c g}\right)\right]=0 \\
& \partial_{t} S_{g}+v_{T} \partial_{x} f_{g}-\partial_{x}\left[\frac{k}{\phi}\left(-\lambda_{g} f_{w} \partial_{x} P_{c w}+\lambda_{g}\left(1-f_{g}\right) \partial_{x} P_{c g}\right)\right]=0
\end{aligned}
$$




\section{Remarks.}

1. The mathematical problem is complete up to imposition of initial and boundary conditions, and definition of the relative permeability and capillary pressure functions.

2. The pressure equation (11) and the system of saturation equations (17)(18) are coupled through the capillary pressures.

3. This coupling is of little consequence in one-dimensional models, because the solution to the pressure equation is trivial in this case. The total velocity is at most a function of time and, therefore, dependent only on the boundary conditions.

4. In the multidimensional case, the pressure and saturation equations may be decoupled by introducing a global pressure [7].

\subsection{Relative permeabilities and capillary pressures}

Relative permeabilities are the key descriptors of classical Darcy-type formulations of multiphase flow through porous media. Strictly speaking, relative permeabilities should depend not only on the fluid saturations, but also on the saturation history, wettability characteristics, gravity effects, and fluid viscosities [43,44]. Thus, they should be properly called functionals, rather than functions. In this paper, however, we shall understand the relative permeabilities as functions of fluid saturations alone. In particular, we shall use relative permeabilities that satisfy Stone's assumptions [60,61], that is, water and gas relative permeabilities depend only on the water and gas saturations, respectively, and oil relative permeability depends of both:

$$
\begin{aligned}
k_{r w} & =k_{r w}\left(S_{w}\right), \\
k_{r o} & =k_{r o}\left(S_{w}, S_{g}\right), \\
k_{r g} & =k_{r g}\left(S_{g}\right) .
\end{aligned}
$$

Similar considerations apply to the capillary pressures. In the context of multiphase displacements, capillarity effects lead to a nonlinear diffusion term, whose role is to smear the moving fronts - shocks - that arise in the displacement process. The detailed structure of these shocks - and consequently the capillary pressures - , should depend on several factors, including wettability properties, viscosity ratios, displacement process (drainage or 
imbibition) and pore-scale fluid configuration [48]. For the purpose of this paper, however, we shall use Leverett's assumption $[3,50]$ that the water and gas capillary pressures depend only on the water and gas saturations, respectively:

$$
\begin{aligned}
P_{c w} & =P_{c w}\left(S_{w}\right), \\
P_{c g} & =P_{c g}\left(S_{g}\right) .
\end{aligned}
$$

\subsection{Equations in dimensionless form}

It is convenient, both for a mathematical and a numerical analysis, to write the governing equations (17)-(18) in dimensionless form. We define the dimensionless space and time coordinates:

$$
\begin{aligned}
x_{D} & :=\frac{x}{L} \\
t_{D} & :=\frac{1}{L} \int_{0}^{t} v_{T}(\tau) \mathrm{d} \tau,
\end{aligned}
$$

respectively. We define also the water and gas capillary numbers:

$$
\begin{aligned}
C_{w} & :=\frac{(k / \phi) P_{c w}^{*}}{v_{T} \mu_{w} L}, \\
C_{g} & :=\frac{(k / \phi) P_{c g}^{*}}{v_{T} \mu_{g} L},
\end{aligned}
$$

where $P_{c w}^{*}$ and $P_{c g}^{*}$ are characteristic values of the water and gas capillary pressures, e.g.,

$$
\begin{aligned}
P_{c w}^{*} & :=\int_{0}^{1}\left|P_{c w}(s)\right| \mathrm{d} s, \\
P_{c g}^{*} & :=\int_{0}^{1}\left|P_{c g}(s)\right| \mathrm{d} s .
\end{aligned}
$$

Dimensionless water and gas capillary pressures are defined as follows:

$$
\begin{aligned}
P_{c w}^{D} & :=\frac{P_{c w}}{P_{c w}^{*}}, \\
P_{c g}^{D} & :=\frac{P_{c g}}{P_{c g}^{*}} .
\end{aligned}
$$


Using the definitions above, and the fact that the total velocity $v_{T}$ is at most a function of time, the system of saturation equations may be written as:

$$
\begin{aligned}
& \frac{\partial S_{w}}{\partial t_{D}}+\frac{\partial f_{w}}{\partial x_{D}}-\frac{\partial}{\partial x_{D}}\left[C_{w} \mu_{w} \lambda_{w}\left(1-f_{w}\right) \frac{\partial P_{c w}^{D}}{\partial x_{D}}-C_{g} \mu_{g} \lambda_{w} f_{g} \frac{\partial P_{c g}^{D}}{\partial x_{D}}\right]=0 \\
& \frac{\partial S_{g}}{\partial t_{D}}+\frac{\partial f_{g}}{\partial x_{D}}-\frac{\partial}{\partial x_{D}}\left[-C_{w} \mu_{w} \lambda_{g} f_{w} \frac{\partial P_{c w}^{D}}{\partial x_{D}}+C_{g} \mu_{g} \lambda_{g}\left(1-f_{g}\right) \frac{\partial P_{c g}^{D}}{\partial x_{D}}\right]=0
\end{aligned}
$$

Using the Leverett assumption (20) on the capillary pressures, and defining

$$
\epsilon_{w}:=C_{w} \mu_{w}, \quad \epsilon_{g}:=C_{g} \mu_{g}
$$

we write the system in the equivalent form:

$$
\begin{aligned}
& \frac{\partial S_{w}}{\partial t_{D}}+\frac{\partial f_{w}}{\partial x_{D}}-\frac{\partial}{\partial x_{D}}\left[\epsilon_{w} \lambda_{w}\left(1-f_{w}\right) \frac{\mathrm{d} P_{c w}^{D}}{\mathrm{~d} S_{w}} \frac{\partial S_{w}}{\partial x_{D}}-\epsilon_{g} \lambda_{w} f_{g} \frac{\mathrm{d} P_{c g}^{D}}{\mathrm{~d} S_{g}} \frac{\partial S_{g}}{\partial x_{D}}\right]=0 \\
& \frac{\partial S_{g}}{\partial t_{D}}+\frac{\partial f_{g}}{\partial x_{D}}-\frac{\partial}{\partial x_{D}}\left[-\epsilon_{w} \lambda_{g} f_{w} \frac{\mathrm{d} P_{c w}^{D}}{\mathrm{~d} S_{w}} \frac{\partial S_{w}}{\partial x_{D}}+\epsilon_{g} \lambda_{g}\left(1-f_{g}\right) \frac{\mathrm{d} P_{c g}^{D}}{\mathrm{~d} S_{g}} \frac{\partial S_{g}}{\partial x_{D}}\right]=0
\end{aligned}
$$

Understanding the independent variables $x$ and $t$ as their dimensionless counterparts, we write the saturation equations in their final form as a nonlinear system of conservation laws:

$$
\partial_{t} \boldsymbol{u}+\partial_{x} \boldsymbol{f}-\partial_{x}\left(\mathbf{D} \partial_{x} \boldsymbol{u}\right)=\mathbf{0}
$$

where

$$
\begin{aligned}
\boldsymbol{u} & :=\left(\begin{array}{c}
S_{w} \\
S_{g}
\end{array}\right), \\
\boldsymbol{f} & :=\left(\begin{array}{c}
f_{w} \\
f_{g}
\end{array}\right), \\
\mathbf{D} & :=\left(\begin{array}{ll}
D_{w w} & D_{w g} \\
D_{g w} & D_{g g}
\end{array}\right)=\left(\begin{array}{cc}
\epsilon_{w} \lambda_{w}\left(1-f_{w}\right) \frac{\mathrm{d} P_{c w}^{D}}{\mathrm{~d} S_{w}} & -\epsilon_{g} \lambda_{w} f_{g} \frac{\mathrm{d} P_{c g}^{D}}{\mathrm{~d} S_{g}} \\
-\epsilon_{w} \lambda_{g} f_{w} \frac{\mathrm{d} P_{c w}^{D}}{\mathrm{~d} S_{w}} & \epsilon_{g} \lambda_{g}\left(1-f_{g}\right) \frac{\mathrm{d} P_{c g}^{D}}{\mathrm{~d} S_{g}}
\end{array}\right),
\end{aligned}
$$

are the vector of unknown saturations, the fractional flow vector and the capillary-diffusion tensor, respectively. 


\section{Remarks.}

1. The fractional flow and the diffusion tensor are (nonlinear) functions of the unknown saturations, i.e.,

$$
\boldsymbol{f}=\boldsymbol{f}(\boldsymbol{u}), \quad \mathbf{D}=\mathbf{D}(\boldsymbol{u})
$$

2. Under a (linear) change of variables, the water and gas saturations may be understood as normalized saturations, rather than actual saturations [43]. In what follows we shall understand that this change of variables has been employed, so that the three-phase flow region - the range of saturations where relative permeabilities are strictly positivecovers the entire saturation triangle:

$$
\mathcal{T}:=\left\{\left(S_{w}, S_{g}\right): S_{w} \geq 0, \quad S_{g} \geq 0, \quad S_{w}+S_{g} \leq 1\right\}
$$

The saturation triangle is usually represented as a ternary diagram [43], in which the pair $\left(S_{w}, S_{g}\right)$ corresponds to the triple $\left(S_{w}, S_{g}, S_{o}\right)$, where $S_{o} \equiv 1-S_{w}-S_{g}$ (see Figure 1$)$.

3. The character of the system (34) depends on the eigenvalues and eigenvectors of the Jacobian matrix $\boldsymbol{f}^{\prime}$. In [43] we argue that this matrix must have real and distinct eigenvalues for the solution to be physically plausible, and we derive conditions on the relative permeability functions so that this requirement is satisfied. Here, we further assume that the capillary diffusion tensor is positive semi-definite. Under these conditions, the system of equations is parabolic, and strictly hyperbolic in the limit of vanishing diffusion [66].

\section{Multiscale numerical formulation}

In this section, we describe a multiscale formulation for the numerical solution of the system (34). We are interested in the case of small diffusion, for which the solution develops sharp features (shocks and boundary layers). The multiscale approach leads naturally to a stabilized numerical method, which enhances the stability of the solution, without compromising its accuracy in the regions where the solution is smooth. 


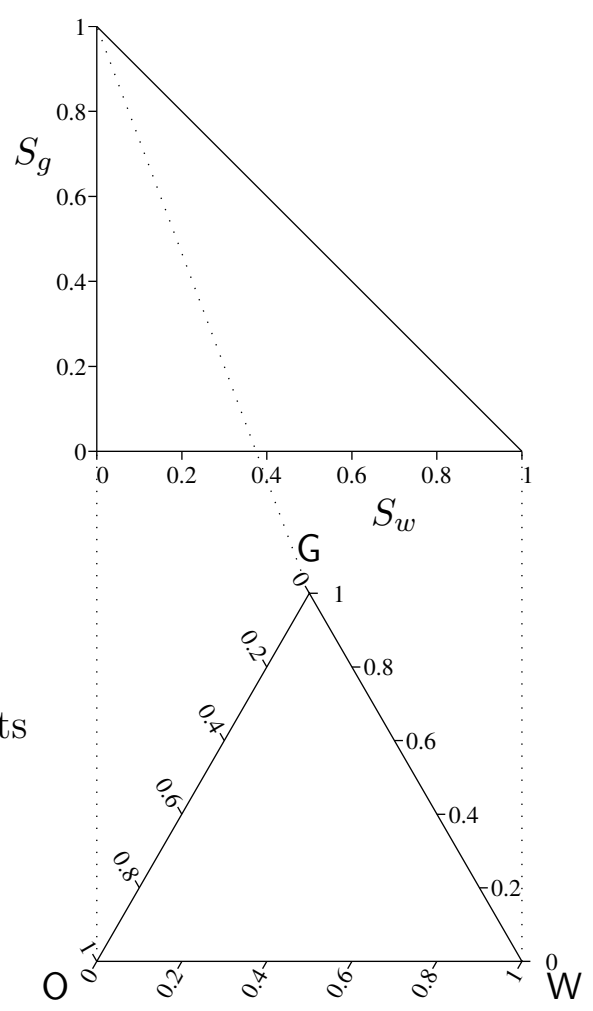

Figure 1. Saturation triangle (top) and ternary diagram (bottom).

The multiple-scale formalism was first proposed in [26], and it is now recognized as a state-of-the-art method in computational fluid dynamics. In $[41,42]$, the formulation was applied to miscible flow of two components - described by a linear advection-diffusion equation-, and immiscible flow of two phases - which is modeled by a nonlinear scalar conservation law-. Here we apply — and extend - the formulation to the problem of three-phase porous media flow.

\subsection{Initial and boundary value problem}

The mathematical problem is defined by the one-dimensional system of conservation laws

$$
\partial_{t} \boldsymbol{u}+\partial_{x}\left(\boldsymbol{f}(\boldsymbol{u})-\mathbf{D}(\boldsymbol{u}) \partial_{x} \boldsymbol{u}\right)=\mathbf{0}, \quad x \in \Omega \equiv(0,1), \quad t \in(0, T]
$$


together with appropriate initial and boundary conditions. We shall consider Dirichlet (essential) and Neumann (natural) boundary conditions. Let $\partial \Omega \equiv$ $\{0,1\}$ be the boundary of the domain, $\Gamma_{u} \subset \partial \Omega$ is the part of the boundary where essential conditions are imposed, and $\Gamma_{n} \equiv \partial \Omega \backslash \Gamma_{u}$ is the part of the boundary with natural boundary conditions:

$$
\begin{aligned}
\boldsymbol{u} & =\overline{\boldsymbol{u}} & & \text { on } \Gamma_{u}, \\
\left(\boldsymbol{f}-\mathbf{D} \partial_{x} \boldsymbol{u}\right) n & =\overline{\boldsymbol{F}} & & \text { on } \Gamma_{n},
\end{aligned}
$$

where $n$ is the outward unit normal to the boundary, i.e., $n=+1$ at $x=1$, and $n=-1$ at $x=0$. The initial conditions

$$
\boldsymbol{u}(x, t=0)=\boldsymbol{u}_{0}(x), \quad x \in \bar{\Omega} \equiv[0,1],
$$

close the definition of the mathematical problem.

\subsection{Weak form}

The development of virtually all integral methods for the numerical solution of the initial and boundary value problem of the previous section starts from the weak form of the mathematical problem. To this end, we define the following functional spaces:

$$
\begin{aligned}
\mathcal{V} & :=\left\{\boldsymbol{v} \in W: \boldsymbol{v}=\overline{\boldsymbol{u}} \text { on } \Gamma_{u}\right\}, \\
\mathcal{V}_{0} & :=\left\{\boldsymbol{v} \in W: \boldsymbol{v}=\mathbf{0} \text { on } \Gamma_{u}\right\},
\end{aligned}
$$

where the appropriate Sobolev space $W$ depends on the particular form of the diffusion tensor. The weak form of problem (40) with boundary and initial conditions (41)-(43) consists in finding $u \in \mathcal{V}$ for each fixed $t \in(0, T]$, such that

$$
\begin{aligned}
\left(\partial_{t} \boldsymbol{u}, \boldsymbol{v}\right)+a(\boldsymbol{u}, \boldsymbol{v} ; \boldsymbol{u}) & =l(\boldsymbol{v}) \quad \forall \boldsymbol{v} \in \mathcal{V}_{0} \\
\boldsymbol{u}(x, t=0) & =\boldsymbol{u}_{0}(x)
\end{aligned}
$$

where

$$
\begin{aligned}
\left(\partial_{t} \boldsymbol{u}, \boldsymbol{v}\right) & =\int_{\Omega} \partial_{t} \boldsymbol{u} \cdot \boldsymbol{v} \mathrm{d} \Omega \\
a(\boldsymbol{u}, \boldsymbol{v} ; \boldsymbol{w}) & =-\int_{\Omega} \boldsymbol{f}(\boldsymbol{w}) \cdot \partial_{x} \boldsymbol{v} \mathrm{d} \Omega+\int_{\Omega} \mathbf{D}(\boldsymbol{w}) \partial_{x} \boldsymbol{u} \cdot \partial_{x} \boldsymbol{v} \mathrm{d} \Omega \\
l(\boldsymbol{v}) & =-\int_{\Gamma_{n}} \overline{\boldsymbol{F}} \cdot \boldsymbol{v} \mathrm{d} \Gamma
\end{aligned}
$$




\section{Remarks.}

1. In the context of classical - smooth - solutions, the strong and weak forms of the mathematical problem are equivalent. The weak form is less restrictive, however, in the sense that it may have weak discontinuous - solutions.

2. The functional spaces $\mathcal{V}$ and $\mathcal{V}_{0}$ are infinite-dimensional.

3. Equation (44) is linear in the test function $\boldsymbol{v}$. This fact is exploited at the implementation level, for it allows to consider test functions of the form:

$$
\boldsymbol{v}=\left(\begin{array}{c}
v_{1} \\
0
\end{array}\right)+\left(\begin{array}{c}
0 \\
v_{2}
\end{array}\right)
$$

\subsection{Classical Galerkin method}

Once the mathematical problem has been stated in weak form, it is straightforward to introduce the classical Galerkin method. Instead of considering the infinite-dimensional spaces $\mathcal{V}$ and $\mathcal{V}_{0}$, one employs conforming finitedimensional spaces $\mathcal{V}_{h} \subset \mathcal{V}$ and $\mathcal{V}_{h, 0} \subset \mathcal{V}_{0}$ of piecewise polynomials, defined on a finite element mesh. The standard Galerkin approximation of (44) reduces to find $\boldsymbol{u}_{h} \in \mathcal{V}_{h}$ for each fixed $t$, such that

$$
\left(\partial_{t} \boldsymbol{u}_{h}, \boldsymbol{v}_{h}\right)+a\left(\boldsymbol{u}_{h}, \boldsymbol{v}_{h} ; \boldsymbol{u}_{h}\right)=l\left(\boldsymbol{v}_{h}\right) \quad \forall \boldsymbol{v}_{h} \in \mathcal{V}_{h, 0},
$$

and $\boldsymbol{u}_{h}(x, t=0)$ is the projection of the initial function $\boldsymbol{u}_{0}(x)$ onto space $\mathcal{V}_{h}$. The system of ordinary differential equations (49) is transformed into a system of (nonlinear) algebraic equations by further discretizing the time derivative [63].

\subsection{Multiple-scale approach}

It is a well-known fact that the classical Galerkin method lacks stability when diffusive effects are exceedingly small, so that the system of equations is nearly hyperbolic. The objective of the multiple-scale approach described here is to obtain a stabilized numerical formulation for this type of problems. The formulation, based on the framework originally introduced in [26] was presented in $[41,42]$ for miscible flow of two components and immiscible 
flow of two phases, which involve scalar conservation laws. The numerical formulation is revisited here, and applied to systems of conservation laws.

The key idea of the formulation is a multiscale split of the variable of interest $\boldsymbol{u} \in \mathcal{V}$ into resolved and unresolved scales:

$$
\boldsymbol{u}=\boldsymbol{u}_{h}+\tilde{\boldsymbol{u}}
$$

where $\boldsymbol{u}_{h}$ is the resolved - grid- scale and $\tilde{\boldsymbol{u}}$ is the unresolved - subgridscale. This decomposition acknowledges that certain components of the solution cannot be captured by the finite element mesh. This fact is especially relevant for advection-dominated problems, where the solution develops sharp features that would require an impractical grid resolution. Decomposition (50) is unique if one can express the original functional space $\mathcal{V}$ as the direct sum of two spaces:

$$
\mathcal{V}=\mathcal{V}_{h} \oplus \tilde{\mathcal{V}}
$$

where $\mathcal{V}_{h}$ is the space of resolved scales and $\tilde{\mathcal{V}}$ is the space of subgrid scales. The space $\tilde{\mathcal{V}}$ is an infinite-dimensional space that completes $\mathcal{V}_{h}$ in $\mathcal{V}$. This space is generally unknown, and it is the role of the subgrid model to provide a successful approximation to it.

The multiscale decomposition was originally proposed for the linear advectiondiffusion equation in [26,27], and then extended to other linear [10,11,23,24, $35,53,55,56]$ and nonlinear $[12,13,19,20,32-34]$ problems. A common approach to deal with nonlinear problems is to linearize the equations upfront, using either a Picard or a Newton strategy $[12,13]$. In this work, however, we resort to the multiscale decomposition prior to any linearization. In the context of nonlinear problems, it seems natural to express the solution at a given iteration step $(k)$ as:

$$
\boldsymbol{u}^{(k)}=\boldsymbol{u}^{(k-1)}+\delta \boldsymbol{u}^{(k-1)} .
$$

The first term on the right hand side should be understood as an approximate solution at the previous iteration level, and the second term as a correction. In principle, both terms are subject to the multiscale decomposition (50):

$$
\begin{gathered}
\boldsymbol{u}^{(k-1)}=\boldsymbol{u}_{h}^{(k-1)}+\tilde{\boldsymbol{u}}^{(k-1)}, \\
\delta \boldsymbol{u}^{(k-1)}=\delta \boldsymbol{u}_{h}^{(k-1)}+\delta \tilde{\boldsymbol{u}}^{(k-1)},
\end{gathered}
$$

Equation (53) requires that the approximate solution $\boldsymbol{u}^{(k-1)}$ is reconstructed after every iteration. To avoid this reconstruction step, and obtain a formulation that completely decouples the resolved and unresolved scales (see 
below), we make the additional approximation:

$$
\boldsymbol{u}^{(k-1)} \approx \boldsymbol{u}_{h}^{(k-1)}
$$

so that the multiscale split takes the form:

$$
\boldsymbol{u}^{(k)} \approx \boldsymbol{u}_{h}^{(k)}+\delta \tilde{\boldsymbol{u}}^{(k-1)}
$$

In what follows we shall drop superscripts referring to the iteration level, and simply write

$$
\boldsymbol{u} \approx \boldsymbol{u}_{h}+\delta \tilde{\boldsymbol{u}}
$$

\section{Remarks.}

1. We refer to Equation (57) as an incremental formulation, with a multiscale decomposition of the increment.

2. The term $\boldsymbol{u}_{h}$ in Equation (57) should be understood as an approximate solution about which the equations are linearized. The term $\delta \tilde{\boldsymbol{u}}$ plays the role of a perturbation that will allow stabilization of the solution.

3. The working assumption (55) makes our formulation different from that in [13] and [19,20], where the subgrid scales are tracked, and the multiscale variable is reconstructed after every step of the iterative process.

The derivation of the multiscale formulation starts by invoking a multiscale split of the solution $\boldsymbol{u}$ and the test function $\boldsymbol{v}$ :

$$
\begin{aligned}
& \boldsymbol{u}=\boldsymbol{u}_{h}+\delta \tilde{\boldsymbol{u}} \in \mathcal{V}=\mathcal{V}_{h} \oplus \tilde{\mathcal{V}} \\
& \boldsymbol{v}=\boldsymbol{v}_{h}+\tilde{\boldsymbol{v}} \in \mathcal{V}_{0}=\mathcal{V}_{h, 0} \oplus \tilde{\mathcal{V}}
\end{aligned}
$$

Because the weak form is linear with respect to the test function $\boldsymbol{v}$, the original mathematical problem (44) is split into two, a grid scale problem:

$$
\left(\partial_{t}\left(\boldsymbol{u}_{h}+\delta \tilde{\boldsymbol{u}}\right), \boldsymbol{v}_{h}\right)+a\left(\boldsymbol{u}_{h}+\delta \tilde{\boldsymbol{u}}, \boldsymbol{v}_{h} ; \boldsymbol{u}_{h}+\delta \tilde{\boldsymbol{u}}\right)=l\left(\boldsymbol{v}_{h}\right) \quad \forall \boldsymbol{v}_{h} \in \mathcal{V}_{h, 0},
$$

and a subscale problem:

$$
\left(\partial_{t}\left(\boldsymbol{u}_{h}+\delta \tilde{\boldsymbol{u}}\right), \tilde{\boldsymbol{v}}\right)+a\left(\boldsymbol{u}_{h}+\delta \tilde{\boldsymbol{u}}, \tilde{\boldsymbol{v}} ; \boldsymbol{u}_{h}+\delta \tilde{\boldsymbol{u}}\right)=l(\tilde{\boldsymbol{v}}) \quad \forall \tilde{\boldsymbol{v}} \in \tilde{\mathcal{V}}
$$

It is important to note that the former is a finite-dimensional problem, whereas the latter is infinite-dimensional. 


\subsubsection{Subgrid scale problem}

Here we derive the final form of the subscale equations, and introduce all the approximations required along the process. We start by writing the flux term as a sum of element integrals, and integrate by parts on each element:

$$
\begin{aligned}
a & \left(\boldsymbol{u}_{h}+\delta \tilde{\boldsymbol{u}}, \tilde{\boldsymbol{v}} ; \boldsymbol{u}_{h}+\delta \tilde{\boldsymbol{u}}\right) \\
= & -\sum_{e} \int_{\Omega^{e}}\left(\boldsymbol{f}\left(\boldsymbol{u}_{h}+\delta \tilde{\boldsymbol{u}}\right)-\mathbf{D}\left(\boldsymbol{u}_{h}+\delta \tilde{\boldsymbol{u}}\right) \partial_{x}\left(\boldsymbol{u}_{h}+\delta \tilde{\boldsymbol{u}}\right)\right) \cdot \partial_{x} \tilde{\boldsymbol{v}} \mathrm{d} \Omega \\
= & \sum_{e} \int_{\Omega^{e}} \partial_{x}\left(\boldsymbol{f}\left(\boldsymbol{u}_{h}+\delta \tilde{\boldsymbol{u}}\right)-\mathbf{D}\left(\boldsymbol{u}_{h}+\delta \tilde{\boldsymbol{u}}\right) \partial_{x}\left(\boldsymbol{u}_{h}+\delta \tilde{\boldsymbol{u}}\right)\right) \cdot \tilde{\boldsymbol{v}} \mathrm{d} \Omega \\
& -\sum_{e} \int_{\Gamma^{e}}\left(\boldsymbol{f}\left(\boldsymbol{u}_{h}+\delta \tilde{\boldsymbol{u}}\right)-\mathbf{D}\left(\boldsymbol{u}_{h}+\delta \tilde{\boldsymbol{u}}\right) \partial_{x}\left(\boldsymbol{u}_{h}+\delta \tilde{\boldsymbol{u}}\right)\right) n \cdot \tilde{\boldsymbol{v}} \mathrm{d} \Gamma
\end{aligned}
$$

We assume continuity of the flux across interelement boundaries, so that the boundary integrals cancel each other on adjacent elements in the interior of the domain, i.e.,

$$
\begin{aligned}
& -\sum_{e} \int_{\Gamma^{e}}\left(\boldsymbol{f}\left(\boldsymbol{u}_{h}+\delta \tilde{\boldsymbol{u}}\right)-\mathbf{D}\left(\boldsymbol{u}_{h}+\delta \tilde{\boldsymbol{u}}\right) \partial_{x}\left(\boldsymbol{u}_{h}+\delta \tilde{\boldsymbol{u}}\right)\right) n \cdot \tilde{\boldsymbol{v}} \mathrm{d} \Gamma \\
& \quad \approx-\int_{\Gamma_{n}} \overline{\boldsymbol{F}} \cdot \tilde{\boldsymbol{v}} \mathrm{d} \Gamma \equiv l(\tilde{\boldsymbol{v}}) .
\end{aligned}
$$

The expression above is a true identity if $\boldsymbol{u}_{h}+\delta \tilde{\boldsymbol{u}}$ is the exact solution, or if locally mass conservative finite element spaces are employed. Otherwise, Equation (63) should be regarded as an approximation.

We now approximate the total flux by a first-order Taylor expansion about the coarse-scale solution $\boldsymbol{u}_{h}$ :

$$
\begin{aligned}
& \boldsymbol{f}\left(\boldsymbol{u}_{h}+\delta \tilde{\boldsymbol{u}}\right)-\mathbf{D}\left(\boldsymbol{u}_{h}+\delta \tilde{\boldsymbol{u}}\right) \partial_{x}\left(\boldsymbol{u}_{h}+\delta \tilde{\boldsymbol{u}}\right) \\
&= \boldsymbol{f}\left(\boldsymbol{u}_{h}\right)-\mathbf{D}\left(\boldsymbol{u}_{h}\right) \partial_{x} \boldsymbol{u}_{h} \\
&+\boldsymbol{f}^{\prime}\left(\boldsymbol{u}_{h}\right) \delta \tilde{\boldsymbol{u}}-\left(\mathbf{D}^{\prime}\left(\boldsymbol{u}_{h}\right) \delta \tilde{\boldsymbol{u}}\right) \partial_{x} \boldsymbol{u}_{h}-\mathbf{D}\left(\boldsymbol{u}_{h}\right) \partial_{x}(\delta \tilde{\boldsymbol{u}})+O\left(|\delta \tilde{\boldsymbol{u}}|^{2}\right) .
\end{aligned}
$$

Equation (64) suggests defining the linearized advection-diffusion operator in conservation form:

$$
\mathcal{L}_{u_{h}} \boldsymbol{v}:=\partial_{x}\left[\boldsymbol{f}^{\prime}\left(\boldsymbol{u}_{h}\right) \boldsymbol{v}-\left(\mathbf{D}^{\prime}\left(\boldsymbol{u}_{h}\right) \boldsymbol{v}\right) \partial_{x} \boldsymbol{u}_{h}-\mathbf{D}\left(\boldsymbol{u}_{h}\right) \partial_{x} \boldsymbol{v}\right]
$$


The operator $\mathcal{L}_{u_{h}} \boldsymbol{v}$ depends in a nonlinear fashion on the approximate coarsescale solution $\boldsymbol{u}_{h}$, but is linear in its argument $\boldsymbol{v}$. We write this operator in the more suggestive (and convenient) form:

$$
\mathcal{L}_{u_{h}} \boldsymbol{v}:=\partial_{x}\left[\mathbf{A}\left(\boldsymbol{u}_{h}\right) \boldsymbol{v}-\mathbf{D}\left(\boldsymbol{u}_{h}\right) \partial_{x} \boldsymbol{v}\right]
$$

where $\mathbf{A}\left(\boldsymbol{u}_{h}\right)$ is a $2 \times 2$ "advection" operator, whose components $A_{i j}\left(\boldsymbol{u}_{h}\right)$ take the following expression:

$$
A_{i j}\left(\boldsymbol{u}_{h}\right):=\frac{\partial f_{i}\left(\boldsymbol{u}_{h}\right)}{\partial u_{h, j}}-\sum_{k} \frac{\partial D_{i k}\left(\boldsymbol{u}_{h}\right)}{\partial u_{h, j}} \partial_{x} u_{h, k} .
$$

Using Equations (63)-(66) in Equation (62), we write the first-order approximation of the flux term in the subgrid scale problem (61) as

$$
\begin{aligned}
a\left(\boldsymbol{u}_{h}+\delta \tilde{\boldsymbol{u}}, \tilde{\boldsymbol{v}} ; \boldsymbol{u}_{h}+\delta \tilde{\boldsymbol{u}}\right) \approx & \sum_{e} \int_{\Omega^{e}} \partial_{x}\left(\boldsymbol{f}\left(\boldsymbol{u}_{h}\right)-\mathbf{D}\left(\boldsymbol{u}_{h}\right) \partial_{x} \boldsymbol{u}_{h}\right) \cdot \tilde{\boldsymbol{v}} \mathrm{d} \Omega \\
& +\sum_{e} \int_{\Omega^{e}} \mathcal{L}_{u_{h}} \delta \tilde{\boldsymbol{u}} \cdot \tilde{\boldsymbol{v}} \mathrm{d} \Omega-\int_{\Gamma_{n}} \overline{\boldsymbol{F}} \cdot \tilde{\boldsymbol{v}} \mathrm{d} \Gamma
\end{aligned}
$$

A further approximation is to consider quasi-static subscales [13], i.e.,

$$
\partial_{t} \delta \tilde{\boldsymbol{u}} \approx 0
$$

After this final assumption, and defining the grid-scale residual

$$
\mathcal{R}\left(\boldsymbol{u}_{h}\right):=-\partial_{t} \boldsymbol{u}_{h}-\partial_{x}\left(\boldsymbol{f}\left(\boldsymbol{u}_{h}\right)-\mathbf{D}\left(\boldsymbol{u}_{h}\right) \partial_{x} \boldsymbol{u}_{h}\right),
$$

the subscale problem (61) is written as follows:

$$
\sum_{e} \int_{\Omega^{e}} \mathcal{L}_{u_{h}} \delta \tilde{\boldsymbol{u}} \cdot \tilde{\boldsymbol{v}} \mathrm{d} \Omega=\sum_{e} \int_{\Omega^{e}} \mathcal{R}\left(\boldsymbol{u}_{h}\right) \cdot \tilde{\boldsymbol{v}} \mathrm{d} \Omega \quad \forall \tilde{\boldsymbol{v}} \in \tilde{\mathcal{V}}
$$

Equation (71) illustrates that the subgrid scale problem is in fact a projection problem:

$$
\tilde{\Pi}\left(\mathcal{L}_{u_{h}} \delta \tilde{\boldsymbol{u}}\right)=\tilde{\Pi}\left(\mathcal{R}\left(\boldsymbol{u}_{h}\right)\right)
$$

where $\tilde{\Pi}$ is the $L^{2}$-projection onto the space of subgrid scales $\tilde{\mathcal{V}}$.

\section{Remarks.}


1. The subgrid scale problem is infinite-dimensional, so one cannot expect to solve it exactly. It is necessary to resort to some kind of numerical or analytical approximation.

2. It is finally written in (71) as a sum of volume integrals evaluated elementwise.

3. To reduce dramatically the computational cost of the solution to the subscale problem, it seems appealing to localize the problem, so that it can be approximated element-by-element. The difficulty of this step stems from the fact that the boundary conditions of the local problem - values of the subscales on the inter-element boundaries - are unknown. A common modeling assumption is to use $\left.\boldsymbol{v}\right|_{\Gamma^{e}}=\mathbf{0}$, i.e., the subscales are bubble functions that vanish on the boundaries of each element $[4,5,16,26]$.

4. An alternative to assumption (69) of quasi-static subscales would be to keep track of the value of the subscales in every element, and evaluate the time derivative $\partial_{t} \delta \tilde{\boldsymbol{u}}[13]$. One of the benefits of the quasistatic subscale approximation - in addition to the simplicity of the formulation - is that long-term numerical solutions do not depend on the time integration strategy or the actual time step.

In this paper we employ an algebraic approximation to the subscales, which leads to an algebraic subgrid scale model (ASGS):

$$
\delta \tilde{\boldsymbol{u}} \approx \boldsymbol{\tau}_{u_{h}} \mathcal{R}\left(\boldsymbol{u}_{h}\right)
$$

where $\boldsymbol{\tau}_{u_{h}}$ is a $2 \times 2$ matrix of algebraic coefficients, which depend not only on the system parameters, but also on the grid scale solution $\boldsymbol{u}_{h}$. This approximation is substantiated by the convergence analysis of the linear case [14]. It can also be justified from an asymptotic Fourier analysis [14], and has proven useful in numerical tests. The matrix $\boldsymbol{\tau}_{u_{h}}$ is known as the matrix of stabilizing coefficients or matrix of intrinsic time scales [29], and has dimensions of time. Its design, which should be ultimately dictated by stability and convergence analysis, is one of the most difficult issues in the development of a stabilized numerical method. Many alternatives have been proposed, some of which are reviewed and succinctly described in Section 3.5. 


\subsubsection{Grid scale problem}

We now retake the grid scale equation (60). As in the previous section, we linearize the flux term with respect to the coarse scale solution $\boldsymbol{u}_{h}$ :

$$
\begin{aligned}
a\left(\boldsymbol{u}_{h}+\delta \tilde{\boldsymbol{u}}, \boldsymbol{v}_{h} ; \boldsymbol{u}_{h}+\delta \tilde{\boldsymbol{u}}\right) \\
=-\int_{\Omega}\left(\boldsymbol{f}\left(\boldsymbol{u}_{h}+\delta \tilde{\boldsymbol{u}}\right)-\mathbf{D}\left(\boldsymbol{u}_{h}+\delta \tilde{\boldsymbol{u}}\right) \partial_{x}\left(\boldsymbol{u}_{h}+\delta \tilde{\boldsymbol{u}}\right)\right) \cdot \partial_{x} \boldsymbol{v}_{h} \mathrm{~d} \Omega \\
=-\int_{\Omega}\left(\boldsymbol{f}\left(\boldsymbol{u}_{h}\right)-\mathbf{D}\left(\boldsymbol{u}_{h}\right) \partial_{x} \boldsymbol{u}_{h}\right) \cdot \partial_{x} \boldsymbol{v}_{h} \mathrm{~d} \Omega \\
\quad-\int_{\Omega}\left(\boldsymbol{f}^{\prime}\left(\boldsymbol{u}_{h}\right) \delta \tilde{\boldsymbol{u}}-\left(\mathbf{D}^{\prime}\left(\boldsymbol{u}_{h}\right) \delta \tilde{\boldsymbol{u}}\right) \partial_{x} \boldsymbol{u}_{h}\right) \cdot \partial_{x} \boldsymbol{v}_{h} \mathrm{~d} \Omega \\
\quad+\int_{\Omega}\left(\mathbf{D}\left(\boldsymbol{u}_{h}\right) \partial_{x} \delta \tilde{\boldsymbol{u}}\right) \cdot \partial_{x} \boldsymbol{v}_{h} \mathrm{~d} \Omega+O\left(|\delta \tilde{\boldsymbol{u}}|^{2}\right) .
\end{aligned}
$$

The first term in the final expression of (74) is the Galerkin term:

$$
-\int_{\Omega}\left(\boldsymbol{f}\left(\boldsymbol{u}_{h}\right)-\mathbf{D}\left(\boldsymbol{u}_{h}\right) \partial_{x} \boldsymbol{u}_{h}\right) \cdot \partial_{x} \boldsymbol{v}_{h} \mathrm{~d} \Omega=a\left(\boldsymbol{u}_{h}, \boldsymbol{v}_{h} ; \boldsymbol{u}_{h}\right)
$$

Writing the second integral in (74) as a sum of element integrals, and recalling the expression of the linearized "advection" matrix (67), we get:

$$
\begin{aligned}
-\int_{\Omega}\left(\boldsymbol{f}^{\prime}\left(\boldsymbol{u}_{h}\right) \delta \tilde{\boldsymbol{u}}-\left(\mathbf{D}^{\prime}\left(\boldsymbol{u}_{h}\right) \delta \tilde{\boldsymbol{u}}\right) \partial_{x}\right. & \left.\boldsymbol{u}_{h}\right) \cdot \partial_{x} \boldsymbol{v}_{h} \mathrm{~d} \Omega \\
& =\sum_{e} \int_{\Omega^{e}}\left(-\mathbf{A}^{T}\left(\boldsymbol{u}_{h}\right) \partial_{x} \boldsymbol{v}_{h}\right) \cdot \delta \tilde{\boldsymbol{u}} \mathrm{d} \Omega
\end{aligned}
$$

where $\mathbf{A}^{T}$ is the transpose of $\mathbf{A}$, i.e., $A_{i j}^{T}=A_{j i}$. After integration by parts element by element, the third term in (74) is written as

$$
\begin{aligned}
& \int_{\Omega}\left(\mathbf{D}\left(\boldsymbol{u}_{h}\right) \partial_{x} \delta \tilde{\boldsymbol{u}}\right) \cdot \partial_{x} \boldsymbol{v}_{h} \mathrm{~d} \Omega \\
& \quad=-\sum_{e} \int_{\Omega^{e}} \partial_{x}\left(\mathbf{D}^{T}\left(\boldsymbol{u}_{h}\right) \partial_{x} \boldsymbol{v}_{h}\right) \cdot \delta \tilde{\boldsymbol{u}} \mathrm{d} \Omega+\sum_{e} \int_{\Gamma^{e}}\left(\mathbf{D}^{T}\left(\boldsymbol{u}_{h}\right) \partial_{x} \boldsymbol{v}_{h}\right) n \cdot \delta \tilde{\boldsymbol{u}} \mathrm{d} \Gamma
\end{aligned}
$$

Defining the adjoint of the linearized advection-diffusion operator (66),

$$
\mathcal{L}_{u_{h}}^{*} \boldsymbol{v}:=-\mathbf{A}^{T}\left(\boldsymbol{u}_{h}\right) \partial_{x} \boldsymbol{v}-\partial_{x}\left(\mathbf{D}^{T}\left(\boldsymbol{u}_{h}\right) \partial_{x} \boldsymbol{v}\right)
$$


and its associated boundary operator,

$$
\boldsymbol{b}_{u_{h}}^{*} \boldsymbol{v}:=\left(\mathbf{D}^{T}\left(\boldsymbol{u}_{h}\right) \partial_{x} \boldsymbol{v}\right) n,
$$

and substituting (75)-(77) in Equation (74), the flux term of the grid scale equation takes the form:

$$
\begin{aligned}
a\left(\boldsymbol{u}_{h}+\delta \tilde{\boldsymbol{u}}, \boldsymbol{v}_{h} ; \boldsymbol{u}_{h}+\delta \tilde{\boldsymbol{u}}\right)=a\left(\boldsymbol{u}_{h}, \boldsymbol{v}_{h} ; \boldsymbol{u}_{h}\right) \\
\quad+\sum_{e} \int_{\Omega^{e}} \mathcal{L}_{u_{h}}^{*} \boldsymbol{v}_{h} \cdot \delta \tilde{\boldsymbol{u}} \mathrm{d} \Omega+\sum_{e} \int_{\Gamma^{e}} \boldsymbol{b}_{u_{h}}^{*} \boldsymbol{v}_{h} \cdot \delta \tilde{\boldsymbol{u}} \mathrm{d} \Gamma+O\left(|\delta \tilde{\boldsymbol{u}}|^{2}\right) .
\end{aligned}
$$

Substituting the first-order approximation of (80) in (60), and considering quasi-static subscales as before, we obtain the final form of the grid scale equation:

$$
\begin{aligned}
& \left(\partial_{t} \boldsymbol{u}_{h}, \boldsymbol{v}_{h}\right)+a\left(\boldsymbol{u}_{h}, \boldsymbol{v}_{h} ; \boldsymbol{u}_{h}\right) \\
& +\sum_{e} \int_{\Omega^{e}} \mathcal{L}_{u_{h}}^{*} \boldsymbol{v}_{h} \cdot \delta \tilde{\boldsymbol{u}} \mathrm{d} \Omega+\sum_{e} \int_{\Gamma^{e}} \boldsymbol{b}_{u_{h}}^{*} \boldsymbol{v}_{h} \cdot \delta \tilde{\boldsymbol{u}} \mathrm{d} \Gamma=l\left(\boldsymbol{v}_{h}\right) \quad \forall \boldsymbol{v}_{h} \in \mathcal{V}_{h, 0} .
\end{aligned}
$$

\section{Remarks.}

1. By direct comparison with (49), it is immediate to identify in Equation (81) the Galerkin terms and the additional stabilizing terms of the multiscale formulation.

2. The stabilizing terms are evaluated element by element, and consist of a volume integral and a boundary integral. The boundary contribution to the stabilizing term is neglected in the numerical simulations of Section 4. This simplification is sensible only if the magnitude of the diffusive effects is small, which is precisely the case of interest.

3. The grid scale equation (81) and the subgrid scale equation (71) are coupled through the value of the subscales $\delta \tilde{\boldsymbol{u}}$. For the simple subgrid scale model employed here, the algebraic approximation (73) is substituted in Equation (81).

4. Because the subscales are proportional to the grid-scale residual Equation (73) - , the formulation is residual-based and, therefore, automatically consistent. 
5. The novel features of our formulation are the following:

(a) Linearization of the equations is employed after the multiscale split. In particular, only the subscale effects are linearized, whereas the full nonlinear Galerkin term is retained in the grid scale equation.

(b) The approximate solution is not reconstructed after every step in the iterative process, or even after every time step. The benefit of this working assumption is that subscale effects enter the formulation in an integral sense only.

\subsection{Matrix of stabilizing coefficients}

The description of the multiscale finite element formulation is complete up to the definition of the matrix of stabilizing coefficients $\boldsymbol{\tau}_{u_{h}}$. The design of this matrix is one of the most difficult - and controversial - issues in the development of a stabilized formulation. This modeling step is not specific to the multiscale formulation explained here and, in fact, is shared by other stabilized formulations such as Streamline Upwind Petrov-Galerkin (SUPG) [6] and Galerkin least-squares (GLS) [28]. Here we review briefly several options that have been considered in the literature. They all define the stabilization matrix $\boldsymbol{\tau}$ for the linear advection-diffusion problem. Extension to the nonlinear problem is straightforward, after defining the linearized advectiondiffusion operator (66).

\subsubsection{Definition through an eigenvalue problem}

This formulation was originally developed in [29]. The idea is to start from the formulation of the scalar one-dimensional linear advection-diffusion equation [6] - for which it is possible to define a function $\tau$ so that the numerical solution is nodally exact - and extend it to systems of equations in multidimensions. The basis for such extension is to diagonalize the system of equations, by solving an eigenvalue problem, and transform the matrix of stabilizing coefficients accordingly.

Consider the one-dimensional $n \times n$ system of linear advection-diffusion equations:

$$
\partial_{t} \boldsymbol{u}+\mathcal{L} \boldsymbol{u} \equiv \partial_{t} \boldsymbol{u}+\partial_{x}\left(\mathbf{A} \boldsymbol{u}-\mathbf{D} \partial_{x} \boldsymbol{u}\right)=\mathbf{0}
$$


where the diffusion matrix is proportional to the identity matrix, $\mathbf{D}=\epsilon \mathbf{1}$, and the advection matrix $\mathbf{A}$ is assumed to be diagonalizable and have real eigenvalues. Let $\nu_{i}$ and $\boldsymbol{r}_{i}$ be the eigenvalues and eigenvectors of matrix $\mathbf{A}$, i.e.,

$$
\left(\mathbf{A}-\nu_{i} \mathbf{1}\right) \boldsymbol{r}_{i}=\mathbf{0}, \quad i=1, \ldots, n .
$$

We further define the $n \times n$ matrices of eigenvalues and right eigenvectors:

$$
\boldsymbol{\Lambda}:=\operatorname{diag}\left(\nu_{1}, \ldots, \nu_{n}\right), \quad \mathbf{R}:=\left[\boldsymbol{r}_{1}, \ldots, \boldsymbol{r}_{n}\right]
$$

The following identities are used throughout:

$$
\mathbf{\Lambda} \equiv \mathbf{R}^{T} \mathbf{A R}, \quad \mathbf{R}^{T} \mathbf{R} \equiv \mathbf{R}^{T} \equiv \mathbf{1}
$$

The change of variables

$$
\boldsymbol{u}=\mathbf{R} \hat{\boldsymbol{u}}
$$

can be used to diagonalize (82):

$$
\partial_{t} \hat{\boldsymbol{u}}+\hat{\mathcal{L}} \hat{\boldsymbol{u}} \equiv \partial_{t} \hat{\boldsymbol{u}}+\partial_{x}\left(\boldsymbol{\Lambda} \hat{\boldsymbol{u}}-\epsilon \partial_{x} \hat{\boldsymbol{u}}\right)=\mathbf{0}
$$

The system (87) is uncoupled, and the $i$-th equation is a scalar linear advectiondiffusion equation:

$$
\partial_{t} \hat{u}_{i}+\partial_{x}\left(\nu_{i} \hat{u}_{i}-\epsilon \partial_{x} \hat{u}_{i}\right)=0 \text {. }
$$

The goal is to define a stabilization matrix $\boldsymbol{\tau}$ that treats each one of the scalar equations (88) in an optimal fashion.

Following [11], the grid scale equation of the linear problem (82) can be written, after neglecting the contribution from the interelement boundary integrals, in the following convenient form (subscript $h$ referring to the coarse scale has been omitted):

$$
\int_{\Omega} \partial_{t} \boldsymbol{u} \cdot \boldsymbol{v} \mathrm{d} \Omega+\int_{\Omega} \mathcal{L} \boldsymbol{u} \cdot \boldsymbol{v} \mathrm{d} \Omega+\int_{\Omega} \mathcal{L}^{*} \boldsymbol{v} \cdot \boldsymbol{\tau} \mathcal{R} \boldsymbol{u} \mathrm{d} \Omega=\mathbf{0},
$$

where the adjoint operator and the grid scale residual are, respectively,

$$
\begin{aligned}
\mathcal{L}^{*} \boldsymbol{v} & =-\mathbf{A}^{T} \partial_{x} \boldsymbol{v}-\partial_{x}\left(\epsilon \partial_{x} \boldsymbol{v}\right), \\
\mathcal{R} \boldsymbol{u} & =-\partial_{t} \boldsymbol{u}-\mathcal{L} \boldsymbol{u}
\end{aligned}
$$


It is understood that the second term in (89) is integrated by parts, and that the third integral - the stabilizing term - is evaluated element by element. Under the change of variables (86), the Galerkin contribution to Equation (89) is:

$$
\begin{aligned}
\int_{\Omega} \partial_{t} \boldsymbol{u} \cdot \boldsymbol{v} \mathrm{d} \Omega+\int_{\Omega} \mathcal{L} \boldsymbol{u} \cdot \boldsymbol{v} \mathrm{d} \Omega & =\int_{\Omega} \mathbf{R} \partial_{t} \hat{\boldsymbol{u}} \cdot \boldsymbol{v} \mathrm{d} \Omega+\int_{\Omega} \mathbf{R} \hat{\mathcal{L}} \hat{\boldsymbol{u}} \cdot \boldsymbol{v} \mathrm{d} \Omega \\
& =\int_{\Omega} \partial_{t} \hat{\boldsymbol{u}} \cdot \mathbf{R}^{T} \boldsymbol{v} \mathrm{d} \Omega+\int_{\Omega} \hat{\mathcal{L}} \hat{\boldsymbol{u}} \cdot \mathbf{R}^{T} \boldsymbol{v} \mathrm{d} \Omega
\end{aligned}
$$

Therefore, the Galerkin contribution behaves correctly under the change of variables (86) if the test function transforms as $\hat{\boldsymbol{v}}=\mathbf{R}^{T} \boldsymbol{v}$ or, equivalently,

$$
\boldsymbol{v}=\mathbf{R} \hat{\boldsymbol{v}}
$$

We now analyze the stabilizing term of the multiscale formulation. It is not difficult to show that, under the change of variables (86) and (93),

$$
\begin{aligned}
\mathcal{L}^{*} \boldsymbol{v} & =\mathbf{R} \hat{\mathcal{L}}^{*} \hat{\boldsymbol{v}} \\
\mathcal{R} \boldsymbol{u} & =\mathbf{R} \hat{\mathcal{R}} \hat{\boldsymbol{u}} .
\end{aligned}
$$

Using (94)-(95), the stabilizing term in Equation (89) is:

$$
\int_{\Omega} \mathcal{L}^{*} \boldsymbol{v} \cdot \boldsymbol{\tau} \mathcal{R} \boldsymbol{u} \mathrm{d} \Omega=\int_{\Omega} \hat{\mathcal{L}}^{*} \hat{\boldsymbol{v}} \cdot \underbrace{\mathbf{R}^{T} \boldsymbol{\tau} \mathbf{R}}_{=: \hat{\boldsymbol{\tau}}} \hat{\mathcal{R}} \hat{\boldsymbol{u}} \mathrm{d} \Omega .
$$

We conclude that the proper definition of the matrix of stabilizing coefficients $\boldsymbol{\tau}$ for the ASGS formulation of problem (82) is

$$
\boldsymbol{\tau}=\mathbf{R} \hat{\tau} \mathbf{R}^{T}
$$

where

$$
\hat{\boldsymbol{\tau}}=\operatorname{diag}\left(\hat{\tau}_{1}, \ldots, \hat{\tau}_{n}\right),
$$

and each intrinsic time $\hat{\tau}_{i}$ is defined for the corresponding scalar equation of the - uncoupled - system (87). The optimal definition of the intrinsic time when linear finite elements are used - which is nodally exact for the steady-state advection-diffusion equation [6] - takes the form:

$$
\hat{\tau}_{i}=\frac{1}{2} h \frac{\hat{\xi}\left(\alpha_{i}\right)}{\left|\nu_{i}\right|}
$$


where $h$ is the size of the element, $\alpha_{i}$ is a measure of the element Peclet number for the $i$-th equation,

$$
\alpha_{i}=\frac{1}{2} \frac{\left|\nu_{i}\right| h}{\epsilon}
$$

and $\hat{\xi}$ is a diffusion correction factor given by:

$$
\hat{\xi}(\alpha)=\operatorname{coth}(\alpha)-\frac{1}{\alpha} \text {. }
$$

\section{Remarks.}

1. When the system (82) has a diffusion matrix $\mathbf{D}$ that is not proportional to the identity, it is not possible, in general, to fully diagonalize the system. In this case, the pseudo-diagonal system after the change of variables (86) reads:

$$
\partial_{t} \hat{\boldsymbol{u}}+\partial_{x}\left(\boldsymbol{\Lambda} \hat{\boldsymbol{u}}-\mathbf{R}^{T} \mathbf{D R} \partial_{x} \hat{\boldsymbol{u}}\right)=\mathbf{0} .
$$

The equation above suggests using a different diffusion coefficient $\epsilon_{i}$ for each componential Peclet number in Equation (100), given by:

$$
\epsilon_{i}=\boldsymbol{r}_{i}^{T} \mathbf{D} \boldsymbol{r}_{i}
$$

2. Equation (101) provides the optimal diffusion-correction factor, in the sense that the numerical solution to the steady-state diagonalizable advection-diffusion system is nodally exact. However, alternative definitions are possible, which differ in their order of accuracy [59]:

$$
\begin{aligned}
& \hat{\xi}_{0}(\alpha)=1 \\
& \hat{\xi}_{1}(\alpha)=\min (\alpha / 3,1), \\
& \hat{\xi}_{2}(\alpha)=\sqrt{\alpha^{2} /\left(1+\alpha^{2}\right)} \\
& \hat{\xi}_{4}(\alpha)=\sqrt{\alpha^{2} /\left(9+\alpha^{2}\right)} .
\end{aligned}
$$

A graphical comparison of the different definitions of the diffusion correction factor $\hat{\xi}$ as a function of the element Peclet number $\alpha$ is shown in Figure 2. 


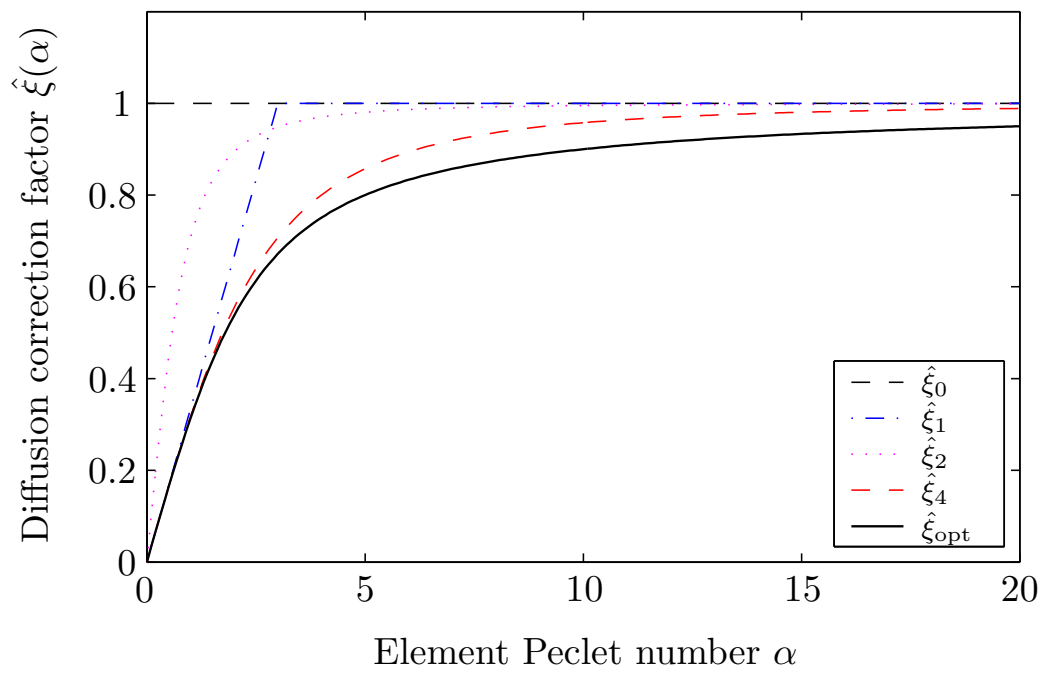

Figure 2. Comparison of alternative definitions of the diffusion correction factor $\hat{\xi}$-Equations (101) and (104)-(107) - as a function of the element Peclet number $\alpha$ (after Shakib et al. [59]).

3. The formulation of the matrix $\boldsymbol{\tau}$ described here applies also to the nonlinear advection-diffusion operator (66). In this case, the advection and diffusion matrices are not constant, but functions of the solution itself. Therefore, the matrix of stabilizing coefficients depends not only on the system parameters but also on the grid scale solution $\boldsymbol{u}_{h}$ :

$$
\boldsymbol{\tau}_{u_{h}}=\mathbf{R}_{u_{h}} \hat{\boldsymbol{\tau}}_{u_{h}} \mathbf{R}_{u_{h}}^{T}
$$

where

$$
\mathbf{R}_{u_{h}}=\left[\boldsymbol{r}_{1}\left(\boldsymbol{u}_{h}\right), \ldots, \boldsymbol{r}_{n}\left(\boldsymbol{u}_{h}\right)\right] .
$$

We denote $\nu_{i}\left(\boldsymbol{u}_{h}\right), \boldsymbol{r}_{i}\left(\boldsymbol{u}_{h}\right)$, the eigenvalues and eigenvectors of the advection matrix $\mathbf{A}\left(\boldsymbol{u}_{h}\right)$, and

$$
\hat{\boldsymbol{\tau}}_{u_{h}}=\operatorname{diag}\left(\hat{\tau}_{1}\left(\boldsymbol{u}_{h}\right), \ldots, \hat{\tau}_{n}\left(\boldsymbol{u}_{h}\right)\right),
$$


where

$$
\begin{aligned}
\hat{\tau}_{i}\left(\boldsymbol{u}_{h}\right) & =\frac{1}{2} h \frac{\hat{\xi}\left(\alpha_{i}\left(\boldsymbol{u}_{h}\right)\right)}{\left|\nu_{i}\left(\boldsymbol{u}_{h}\right)\right|}, \\
\alpha_{i}\left(\boldsymbol{u}_{h}\right) & =\frac{1}{2} \frac{\left|\nu_{i}\left(\boldsymbol{u}_{h}\right)\right| h}{\epsilon_{i}\left(\boldsymbol{u}_{h}\right)} \\
\epsilon_{i}\left(\boldsymbol{u}_{h}\right) & =\boldsymbol{r}_{i}\left(\boldsymbol{u}_{h}\right)^{T} \mathbf{D}\left(\boldsymbol{u}_{h}\right) \boldsymbol{r}_{i}\left(\boldsymbol{u}_{h}\right),
\end{aligned}
$$

and $\hat{\xi}(\alpha)$ is the diffusion correction factor given by Equation (101). Since the advection and diffusion matrices now change from point to point, one needs to solve an eigenvalue problem at each integration point.

\subsubsection{Definition through the matrix square root}

A different formulation of the matrix of stabilizing coefficients was presented in [59], in the context of the Galerkin least-squares (GLS) method. The definition of $\boldsymbol{\tau}$ proposed in [59] is very general and will not be presented here. It suffices to say that it is derived in the framework of a space-time formulation of unsteady multidimensional problems with a Riemannian metric, and includes advection, diffusion, and reaction matrices. When particularized to stationary one-dimensional systems of advection-diffusion type, discretized with linear finite elements, the matrix of stabilizing coefficients takes the form:

$$
\boldsymbol{\tau}_{u_{h}}=\left(\left(\frac{c_{1}}{h^{2}} \mathbf{D}\left(\boldsymbol{u}_{h}\right)\right)^{2}+\left(\frac{c_{2}}{h} \mathbf{A}\left(\boldsymbol{u}_{h}\right)\right)^{2}\right)^{-1 / 2}
$$

where $c_{1}=4$ and $c_{2}=2$.

\section{Remarks.}

1. The definition of $\boldsymbol{\tau}$ given by (114) involves the square-root inverse, which can be computed by exploiting the Cayley-Hamilton theorem (see [52]), by solving an eigenvalue problem, or iteratively by resorting to some kind of Newton's method [59].

2. The matrix $\boldsymbol{\tau}$ defined in this way is obviously symmetric.

3. Expression (114) has also been justified in [14] by means of an asymptotic Fourier analysis. 


\subsubsection{Definition through the matrix inverse}

A different design of the matrix of intrinsic time scales was proposed in [11] for systems of advection-diffusion-reaction equations in multidimensions. When restricted to one-dimensional systems of advection-diffusion type, the expression of $\boldsymbol{\tau}_{u_{h}}$ reduces to:

$$
\boldsymbol{\tau}_{u_{h}}=\left(\frac{c_{1}}{h^{2}} \mathbf{D}\left(\boldsymbol{u}_{h}\right)+\frac{c_{2}}{h} \mathbf{A}\left(\boldsymbol{u}_{h}\right)\right)^{-1}
$$

where $c_{1}=4$ and $c_{2}=2$ for linear elements. This expression emanates from an analysis of the discrete maximum principle in the scalar, stationary, one-dimensional case $[10,11]$.

\section{Remarks.}

1. The matrix $\boldsymbol{\tau}$ given by (115) is a matrix function of the advection and diffusion matrices, which is the essential requirement for the method to provide optimal stabilization for each individual scalar equation when the system is diagonalized [11].

2. Expression (115) may be viewed as an asymptotic approximation of the previous definition of $\boldsymbol{\tau}$-Equation (114) — in the limit of vanishing diffusion.

\subsection{Shock-capturing techniques}

While the multiscale formulation described above will produce stabilized numerical solutions to the three-phase flow equations (see Section 4), overshoots and undershoots may still remain in the neighborhood of internal and boundary layers. The reason for this localized oscillatory behavior is that the method does not guarantee monotonic solutions. One possibility to enhance the robustness of the stabilized formulation is to incorporate a discontinuitycapturing term, that will further reduce or completely eliminate spurious numerical oscillations.

The basic idea of discontinuity-capturing techniques is to introduce an additional term in the grid scale equation (81), which is also evaluated elementwise, and satisfies the following generic design conditions [30,59]:

1. Consistency, which implies that the operator has to be proportional to the grid scale residual. 
2. Enhanced stability, by providing extra control over the gradient of the numerical solution.

3. Accuracy, by vanishing quickly in the regions where the solution is smooth.

Many of the existing discontinuity-capturing formulations can be expressed as an extra diffusion term [9],

$$
\sum_{e} \int_{\Omega^{e}} \mathbf{D}_{s c}\left(\boldsymbol{u}_{h}\right) \partial_{x} \boldsymbol{u}_{h} \cdot \partial_{x} \boldsymbol{v}_{h} \mathrm{~d} \Omega
$$

where the numerical diffusion tensor $\mathbf{D}_{s c}$ depends on the coarse scale solution. This term leads necessarily to a nonlinear method, even if the underlying equation is linear.

\subsubsection{Classical discontinuity-capturing diffusion}

It is not the purpose of this section to derive existing discontinuity-capturing formulations, and the reader is referred to the vast literature on the topic $[9$, $15,18,30,31,36-39,59,62]$. Here we present four different expressions of the shock-capturing diffusion, which are inspired in the original references, and adapted to quasi-static one-dimensional problems of advection-diffusion type, discretized with linear finite elements.

1. A form of the shock-capturing diffusion based on [18] is regarded in [9] as "canonical form":

$$
\mathbf{D}_{s c, 1}=\frac{1}{2} h \frac{\left|\mathcal{R}\left(\boldsymbol{u}_{h}\right)\right|}{\left|\partial_{x} \boldsymbol{u}_{h}\right|} \mathbf{1}
$$

2. The expression above may result in numerical solutions that are a bit too diffusive. Based on an idea of [30,31], the expression above can be modified as follows:

$$
\mathbf{D}_{s c, 2}=\max \left(\frac{1}{2} h \frac{\left|\mathcal{R}\left(\boldsymbol{u}_{h}\right)\right|}{\left|\partial_{x} \boldsymbol{u}_{h}\right|}-\frac{\left|\mathcal{R}\left(\boldsymbol{u}_{h}\right)\right|_{\tau_{u_{h}}}^{2}}{\left|\partial_{x} \boldsymbol{u}_{h}\right|^{2}}, 0\right) \mathbf{1}
$$

where the $\tau$-norm is defined as

$$
|\varphi|_{\tau}^{2}:=\varphi \cdot \tau \varphi
$$


3. A similar expression to (117) above is proposed in [59] (termed "linear form"), and it is based on an extension of the formulation in [30]. A restricted version of it reads:

$$
\mathbf{D}_{s c, 3}=\frac{1}{2} h \frac{\left|\mathcal{R}\left(\boldsymbol{u}_{h}\right)\right|_{\tau_{u_{h}}}}{\left|\partial_{x} \boldsymbol{u}_{h}\right| \tau_{u_{h}}} \mathbf{1}
$$

4. Reference [59] considers also another expression (termed "quadratic form"), which has features in common with the formulation in [18]. When reduced to our one-dimensional model problem, it takes the form:

$$
\mathbf{D}_{s c, 4}=2 \frac{\left|\mathcal{R}\left(\boldsymbol{u}_{h}\right)\right|_{\tau_{u_{h}}}^{2}}{\left|\partial_{x} \boldsymbol{u}_{h}\right|^{2}} \mathbf{1}
$$

All the formulations of the discontinuity-capturing diffusion presented above share several properties, such as being residual-based, dimensionally consistent, and isotropic. We do not discard the possibility that using an anisotropic diffusion tensor formulation would be significantly more effective. This was precisely the conclusion in [9] for the scalar advection-diffusion equation in several space dimensions.

\subsubsection{Novel discontinuity-capturing diffusion}

The motivation for looking into alternatives to the classical formulations of discontinuity-capturing diffusion is that the amount of numerical dissipation introduced by these methods is not sufficiently localized to the neighborhood of shocks and boundary layers. It is easy to understand the reason for this deficiency, by considering the "canonical form" (117) applied to a linear, quasi-steady, advection-dominated, scalar equation. In this case, the grid scale residual is $\mathcal{R}\left(u_{h}\right) \approx-\mathrm{A} \partial_{x} u_{h}$ and, therefore, the shock-capturing diffusion is

$$
\mathrm{D}_{s c, 1} \approx \frac{1}{2} h \frac{\left|-\mathrm{A} \partial_{x} u_{h}\right|}{\left|\partial_{x} u_{h}\right|}=\frac{1}{2} h \mathrm{~A},
$$

which is constant. This means that the formulation introduces the same amount of artificial diffusion everywhere, even though it is only required in the vicinity of sharp gradients [41].

To remedy this undesirable behavior, we propose a discontinuity-capturing diffusion that introduces an essential difference with respect to the classical 
formulations of the previous paragraph: the local gradient $\partial_{x} \boldsymbol{u}_{h}$ is replaced by a global measure of the gradient $\sim \boldsymbol{U}_{s c} / h$. In particular, we shall test the following expression:

$$
\mathbf{D}_{s c, g}=C_{s c} h \frac{\left|\mathcal{R}\left(\boldsymbol{u}_{h}\right)\right|}{\left|\boldsymbol{U}_{s c} / h\right|} \mathbf{1},
$$

where $C_{s c}$ is a constant coefficient. The simulations of the next section clearly show that this formulation introduces numerical diffusion in much narrower regions of the computational domain than classical formulations.

\section{Representative numerical simulations}

In this section we present several simulations of one-dimensional three-phase flow in porous media, as described by the mathematical model of Section 2. For the sole purpose of testing the formulation, the capillary diffusion tensor is taken as a constant isotropic matrix, that is, Equation (37) is replaced by

$$
\mathbf{D}=\left(\begin{array}{cc}
\epsilon_{w} & 0 \\
0 & \epsilon_{g}
\end{array}\right)
$$

As we shall see, the form of the capillary diffusion tensor may affect the detailed structure of individual shocks, but not the shock location and the global structure of the solution. The practical importance of this dependency on the form of the diffusion tensor is minimized by the fact that, because we are interested in the nearly hyperbolic case - which is the most challenging to model numerically - , we shall use very small values of the capillary diffusion coefficients $\epsilon_{w}, \epsilon_{g}$.

The following relative permeability functions are used:

$$
\begin{aligned}
k_{r w} & =S_{w}^{2}, \\
k_{r o} & =\left(1-S_{w}\right)\left(1-S_{g}\right)\left(1-S_{w}-S_{g}\right), \\
k_{r g} & =\beta_{g} S_{g}+\left(1-\beta_{g}\right) S_{g}^{2} .
\end{aligned}
$$

These functions belong to the simple class of functions (19), where the water and gas relative permeabilities depend only on their own saturation, and the oil relative permeability depends on both. The parameter $\beta_{g}$ is the endpoint-slope of the gas relative permeability function. The relevance of this parameter in the context of classical relative permeability models is discussed in $[43,44]$. In the simulations that follow we use the value $\beta_{g}=0.1$. 
Finally, the following values of the fluid viscosity ratios are used:

$$
\frac{\mu_{w}}{\mu_{o}}=0.4375, \quad \frac{\mu_{g}}{\mu_{o}}=0.015
$$

The simulations presented here reproduce the conditions of the Riemann problem, which is an initial value problem on an unbounded domain defined by the system of conservation laws (40), together with piecewise constant initial data separated by a single discontinuity:

$$
\boldsymbol{u}(x, 0)= \begin{cases}\boldsymbol{u}_{l} & \text { if } x<0, \\ \boldsymbol{u}_{r} & \text { if } x>0 .\end{cases}
$$

We model conditions (127) numerically by imposing the initial condition $\boldsymbol{u}(x, 0)=\boldsymbol{u}_{r}$ on a bounded domain $0<x<1$, and a Dirichlet boundary condition $\boldsymbol{u}(0, t)=\boldsymbol{u}_{l}$ on the left boundary. The interest in the Riemann problem is threefold. On one hand, it is particularly challenging to model numerically, since the initial conditions are already discontinuous. Secondly, an analytical solution exists for the capillarity-free case, which can be used to verify the numerical solutions. Finally, it is very valuable in practical applications, because many laboratory and field experiments reproduce in fact the conditions of the Riemann problem.

The general analytical solution to the Riemann problem of capillarityfree three-phase flow is given in $[40,45]$. The system of conservation laws describing three-phase flow is a $2 \times 2$ system, which is strictly hyperbolic for all saturation paths of interest [43]. This implies that there are two separated waves connecting three constant states: $\boldsymbol{u}_{l}$ (left), $\boldsymbol{u}_{m}$ (middle), and $\boldsymbol{u}_{r}$ (right). Therefore, the solution to the Riemann problem of three-phase flow reduces to finding the intermediate constant state $\boldsymbol{u}_{m}$ as the intersection of an admissible 1 -wave $\mathcal{W}_{1}$ (slow wave) and an admissible 2 -wave $\mathcal{W}_{2}$ (fast wave) on the saturation triangle (Figure 3):

$$
\boldsymbol{u}_{l} \stackrel{\mathcal{W}_{1}}{\longrightarrow} \boldsymbol{u}_{m} \stackrel{\mathcal{W}_{2}}{\longrightarrow} \boldsymbol{u}_{r}
$$

Based on the analysis of the wave structure in [40], a wave of the $i$ family connecting two constant states may only be one of the following: an $i$-rarefaction $\left(\mathcal{R}_{i}\right)$, an $i$-shock $\left(\mathcal{S}_{i}\right)$, or an $i$-rarefaction-shock $\left(\mathcal{R}_{i} \mathcal{S}_{i}\right)$. Since the full solution to the Riemann problem is a sequence of two waves, $\mathcal{W}_{1}$ and $\mathcal{W}_{2}$, there are only 9 possible combinations of solutions. A schematic tree with all possible solution types is shown in Figure 4. 


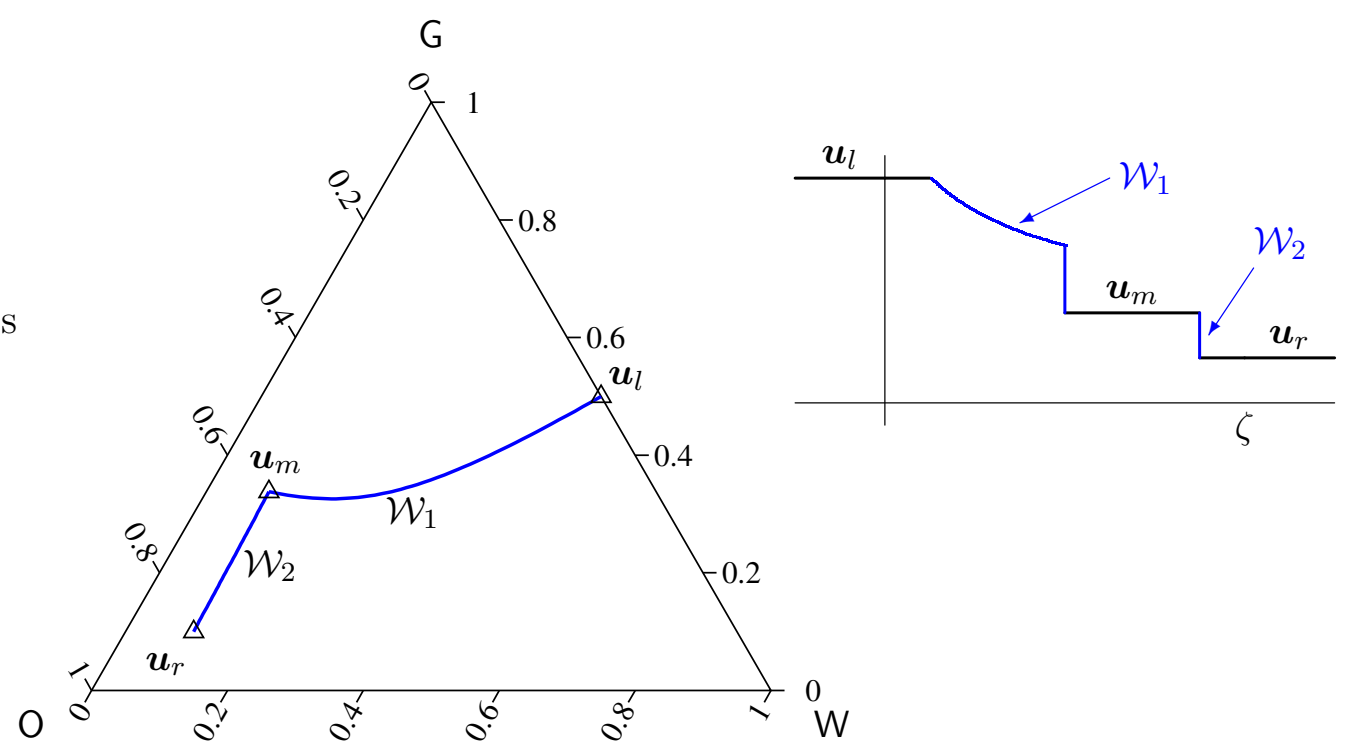

Figure 3. Schematic representation of the generic solution to the Riemann problem of three-phase flow. The solution comprises two distinct waves - slow and fast waves - connecting three constant states. Each wave might involve traveling discontinuities. On the left plot we show a possible configuration of the wave curves in the saturation space. On the right plot we display the corresponding saturation profile for one of the components (gas saturation, say) against the similarity variable $\zeta=x / t$.

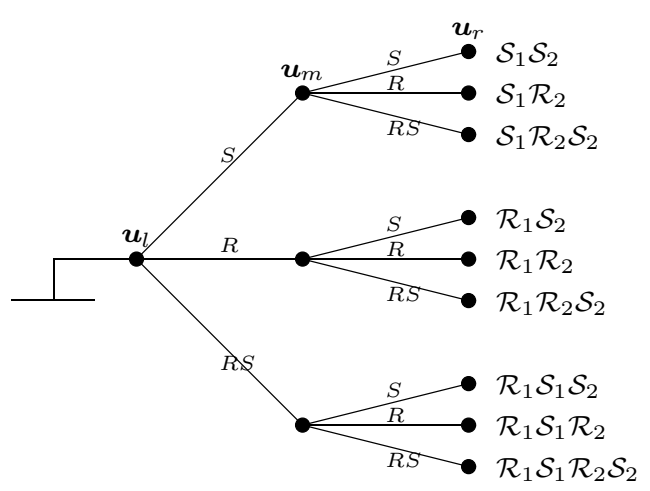

Figure 4. Schematic tree with all possible combinations of solutions to the Riemann problem of three-phase flow. 
Here we study two scenarios: the first one involving oil filtration in a relatively dry soil - which results in a $\mathcal{S}_{1} \mathcal{S}_{2}$ solution-, and the second one reproducing water-gas injection in an oil reservoir - whose solution is of type $\mathcal{R}_{1} \mathcal{S}_{1} \mathcal{S}_{2}$ - Both simulations are transient over a period of time - the solution displays propagating discontinuities - , and then reach quasi-steady conditions - boundary layers are present at the outlet face-. For each of the two problems studied, we compare the exact solution of the capillarityfree model with the numerical solution obtained using the standard Galerkin method on a very fine mesh. Then we compare the performance - on a very coarse mesh - of the classical Galerkin method with the algebraic subgrid scale method, using different formulations for the matrix of stabilizing coefficients. Different discontinuity-capturing formulations are also employed and contrasted. The comparison of stabilized formulations with the standard Galerkin method may seem a little unfair, as the test cases involve nearly hyperbolic systems, for which the classical Galerkin method is known to have unstable behavior. The motivation is to show the stabilizing effect of the new terms in the ASGS formulation, which arise from consideration of the subgrid scales. It is interesting to note that:

1. The ASGS method is in fact a Galerkin method - the coarse-scale trial and test functions belong to the same finite element space. The difference with respect to the classical Galerkin method is that the subgrid scales are modeled separately and incorporated to the coarse scale problem.

2. The computational cost of the ASGS method is essentially the same as that of the standard Galerkin method, as the former involves the calculation of just a few additional integrals, which are evaluated elementwise.

\subsection{Oil filtration in relatively dry soil}

\subsubsection{Description of the problem}

This example reproduces filtration of a mixture of oil, water and gas through a relatively dry porous medium with some water and oil, as shown in Figure 5. The medium has the following initial normalized saturations: $S_{w}=0.15$, $S_{g}=0.8$, and $S_{o}=0.05$. Fluids are injected in a proportion such that the normalized fluid saturations at the inlet face are: $S_{w}=0.25, S_{g}=0.2$, 
Injected saturations

Initial saturations

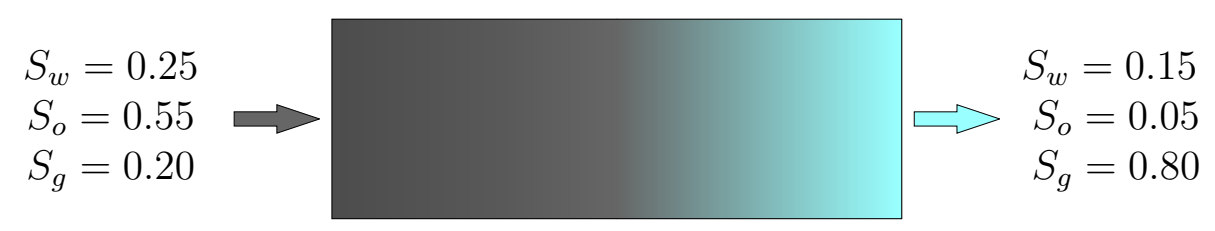

Figure 5. Sketch of the oil filtration problem. A mixture with high oil saturation is injected into a medium initially filled with water and gas.

and $S_{o}=0.55$. Initial saturations are homogeneous on the entire medium, and injected saturations are held constant throughout the experiment, so that the example reproduces the conditions of the Riemann problem. From a practical viewpoint, this problem could represent a contamination event in the shallow subsurface, under one-dimensional flow conditions.

\subsubsection{Analytical solution}

The exact solution to the strictly hyperbolic system of the capillarity-free problem is of type $\mathcal{S}_{1} \mathcal{S}_{2}$, that is, it consists in a sequence of two shocks. Schematically, we denote the structure of the solution as follows:

$$
\boldsymbol{u}_{l} \stackrel{\mathcal{S}_{1}}{\longrightarrow} \boldsymbol{u}_{m} \stackrel{\mathcal{S}_{2}}{\longrightarrow} \boldsymbol{u}_{r}
$$

The left state $\boldsymbol{u}_{l}=\left(S_{w, l}, S_{g, l}\right)=(0.25,0.2)$ corresponds to the injected saturations, and the right state $\boldsymbol{u}_{r}=\left(S_{w, r}, S_{g, r}\right)=(0.15,0.8)$ to the initial saturations. These two states are separated by an intermediate constant state $\boldsymbol{u}_{m}$. The analytical solution may be understood as a slow shock connecting the left and intermediate states, and a fast shock joining the intermediate and right states. Thus, the description of the analytical solution only requires finding the intermediate constant state $\boldsymbol{u}_{m}$ and the speed of propagation $\sigma_{1}$ and $\sigma_{2}$ of the slow and fast shocks, respectively. For the particular data used here, they take the following values:

$$
\boldsymbol{u}_{m}=(0.615,0.328), \quad \sigma_{1}=0.156, \quad \sigma_{2}=0.193
$$

A general and efficient procedure to compute the analytical solution is presented in [40]. The solution - in saturation space - is shown in Figure 6. It 


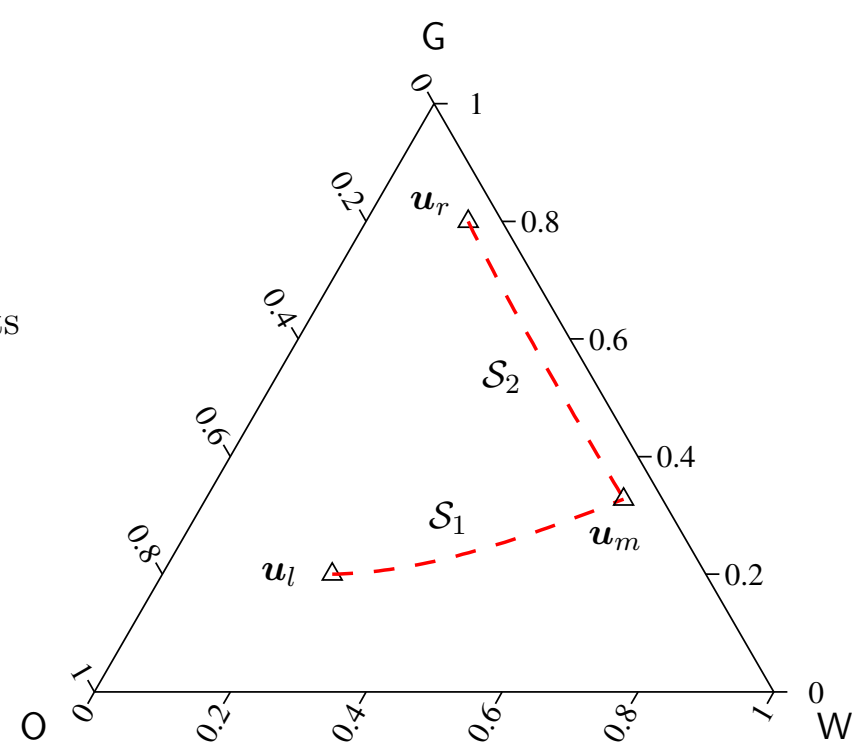

Figure 6. Saturation path of the exact solution to the oil filtration problem. Both waves are shocks $\left(\mathcal{S}_{1} \mathcal{S}_{2}\right.$ solution).

is important to note that dashed lines correspond to the shock curves - set of saturation states that satisfy the Rankine-Hugoniot condition and the Lax entropy condition - , and represent discontinuities in the solution. Therefore, the actual path of the shock curves on the saturation triangle is inconsequential from the point of view of the saturation profiles, and what matters is the location of the endpoints of each shock curve.

In Figure 7 we display in a single plot the profiles of water, gas, and oil saturations against the similarity variable $\zeta=x / t$. The solution at different times can be obtained from one another by simple stretching. Obviously, the solution satisfies constraint (10) that states that the sum of all three saturations adds up to one at all points. The saturations at the right boundary coincide with the initial state, and the saturations at the left boundary correspond to the injected state. This figure clearly illustrates the behavior of the displacement process: basically, the oil phase displaces the water phase, which in turn displaces gas out of the porous medium. One of the key features of the solution is the formation of a water bank - a region where the water saturation is higher than that of the initial and injected states-, so that the solution is not monotonic in the traditional sense. It is also interesting to 


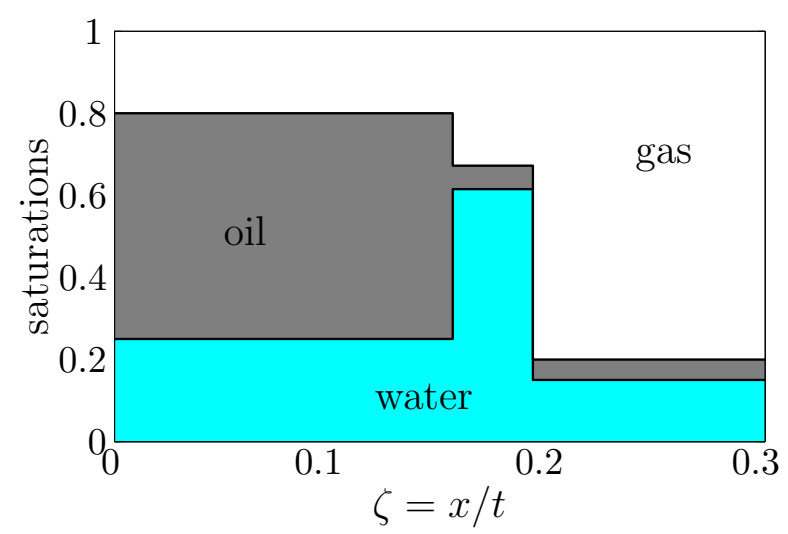

Figure 7. Saturation profiles of the exact solution to the oil filtration problem. Saturations of each phase are plotted against the similarity variable $\zeta=x / t$.

note that the slow shock involves changes in all three saturations, whereas the fast shock connects states with approximately the same oil saturation (see also Figure 6).

\subsubsection{Comparison of numerical solutions}

We test whether the numerical solution to the three-phase oil filtration problem with capillarity provides an accurate approximation to the analytical solution of the capillarity-free case above. Since we are interested in the nearly hyperbolic case, we take small values of the capillary diffusion coefficients in Equation (124):

$$
\epsilon_{w}=0.0005, \quad \epsilon_{g}=0.001 \text {. }
$$

We compute a "reference" numerical solution using the standard Galerkin method on a very fine mesh of 4000 elements. We use a Crank-Nicolson time integration technique with a constant time step of $\delta t=10^{-4}$. Given this discretization and the physical parameters of the problem - in particular the speed of propagation $\sigma_{\max }$ of the fast shock-, we may define the following dimensionless parameters:

$$
\begin{array}{ll}
P e:=\frac{\sigma_{\max } h}{\epsilon_{\min }} \approx 0.1 & \text { (element Peclet number) } \\
C o:=\frac{\sigma_{\max } \delta t}{h} \approx 0.08 & \text { (element Courant number). }
\end{array}
$$




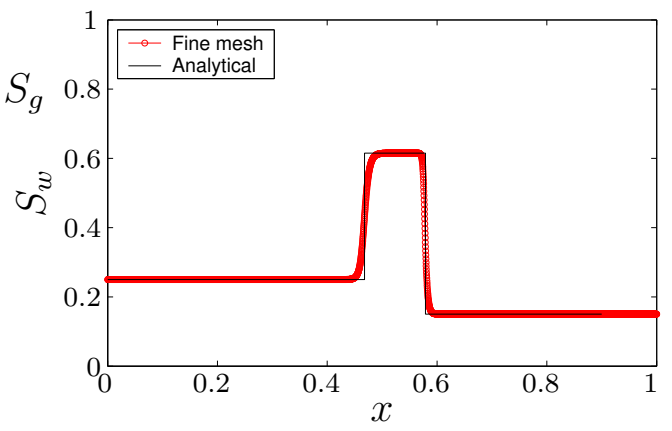

(a) Water saturation

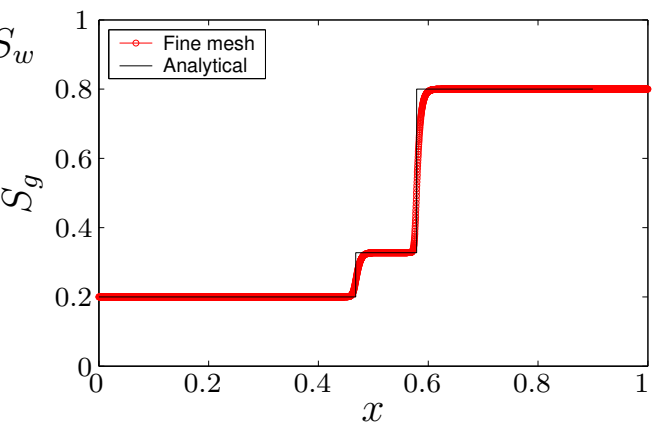

(b) Gas saturation

Figure 8. Saturation profiles of the standard Galerkin solution to the oil filtration problem on a fine mesh of 4000 elements, and comparison to the analytical solution of the capillarity-free case. Results are shown at time $t=3$.

The space and time discretization have been chosen to obtain small values of these two key parameters $(P e \ll 1, C o \ll 1)$, so that the reference solution given by the classical Galerkin method is stable and accurate. The comparison between this solution and the analytical solution described above is presented in Figure 8 at time $t=3$. The "reference" numerical solution captures correctly the global structure of the capillarity-free solution: the location of shocks and the magnitude of the intermediate constant state are predicted accurately. Further numerical simulations - using different values of the capillary diffusion coefficients and different number of elements - confirm that the standard Galerkin solution converges to the entropy solution of the capillarity-free problem. This essential property of the numerical solution - which stems from the fact that the conservation form of the equations is used [49] - is in contrast to the conclusions in [3,64] for two-phase flow, where the nonconservation form of the equation is discretized.

An interesting behavior of the numerical solution is illustrated when the saturation path is plotted on the ternary diagram (Figure 9), where an apparent discrepancy between the numerical and the analytical solutions is readily observed. This "discrepancy" is restricted to the local structure of the fast shock $\mathcal{S}_{2}$ and is, therefore, inconsequential. The detailed structure of the solution around the shock depends in an essential manner on the form of the diffusion tensor, which is ignored in the capillarity-free model and replaced by a discontinuous solution. 


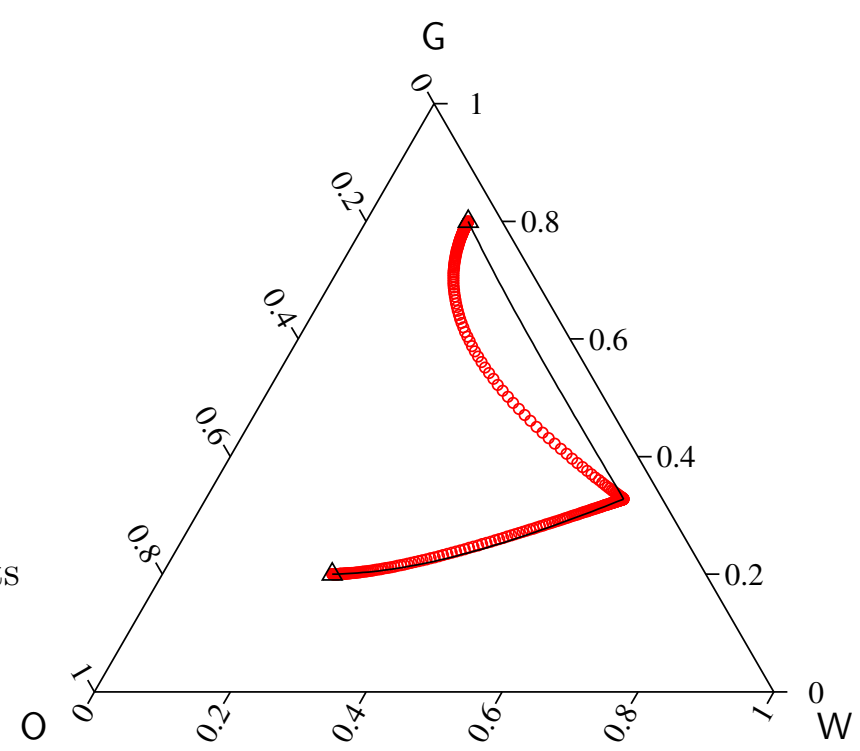

Figure 9. Comparison of the saturation path obtained by the standard Galerkin method on a fine mesh of 4000 elements (dotted curve) and the exact solution (solid curve) of the oil filtration problem.

Standard Galerkin solution. The same problem is solved using the standard Galerkin method on a coarse mesh of only 40 elements. The element Peclet number is now $P e \approx 10$. A Crank-Nicolson time-stepping with $\delta t=0.01$ is used. The associated Courant number is still very small $(C o \approx 0.08)$, to minimize the numerical error introduced by the time discretization. The results of this simulation are shown in Figure 10. The solution obtained with the classical Galerkin method on a fine mesh of 4000 elements is included for reference. Water and gas saturation profiles are plotted at two different times: $t=3$ (transient conditions), and $t=8$ (quasi-steady conditions). It is apparent that the standard Galerkin solution on a coarse grid lacks stability, and is polluted with spurious oscillations. The instabilities are especially severe for the long-term solution, where the oscillatory behavior spreads over most of the computational domain.

Algebraic subgrid scale solutions. We present now the numerical solution to the oil filtration problem obtained with the algebraic subgrid scale (ASGS) method. We recall that the ASGS formulation -Equation (81) - 


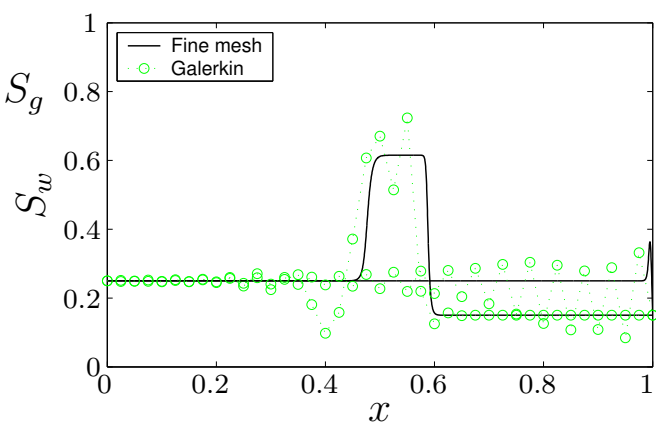

(a) Water saturation

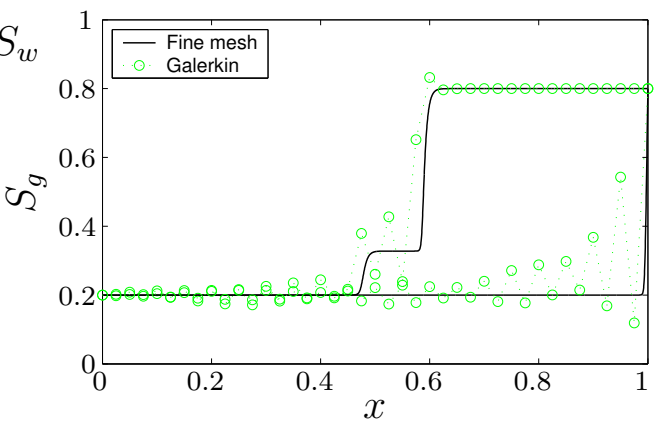

(b) Gas saturation

Figure 10. Saturation profiles of the standard Galerkin solution to the oil filtration problem on a coarse mesh of 40 elements. Results are shown at times $t=3$ and $t=8$.

differs from the classical Galerkin method —Equation (49) — in the addition of a stabilizing term, evaluated element by element. This stabilizing term involves the subgrid scales, which are modeled analytically using an algebraic approximation to the subscales -Equation (73) - . Different alternatives for the definition of the matrix $\boldsymbol{\tau}$ of stabilizing coefficients were discussed in Section 3.5.

In Figure 11 we plot the results obtained with the ASGS method and the definition of $\boldsymbol{\tau}$ given by the eigenvalue problem (108) (formulation proposed by Hughes and Mallet [29]). The solution is much stabler than the standard Galerkin solution. The computed saturation profiles do not display global oscillatory behavior, and capture sharply the transient shocks and the stationary boundary layers. Some small overshoots and undershoots remain, however, but they are confined to the vicinity of the sharp features in the solution.

The ASGS solution obtained with the $\tau$ matrix given by the matrix inverse (115) (formulation proposed by Codina [11]) is shown in Figure 12. The solution is virtually identical to that of Figure 11, and the same comments apply.

Stabilized solutions with shock capturing diffusion. In an attempt to reduce, or completely eliminate, the localized wiggles that remain in the solution of the stabilized ASGS method -Figures 11 and 12 above-, we test several shock-capturing techniques, as described in Section 3.6. We compare 


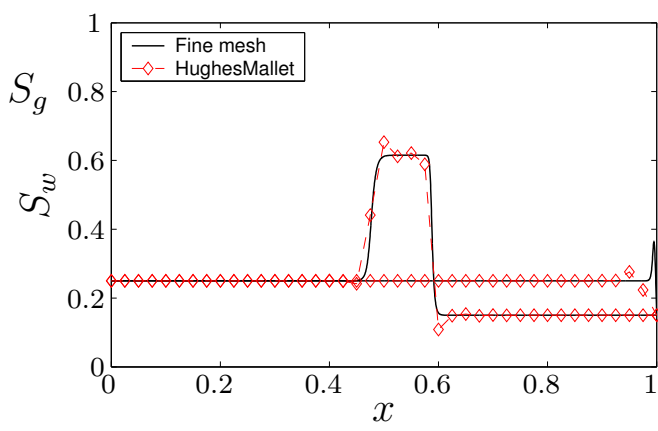

(a) Water saturation

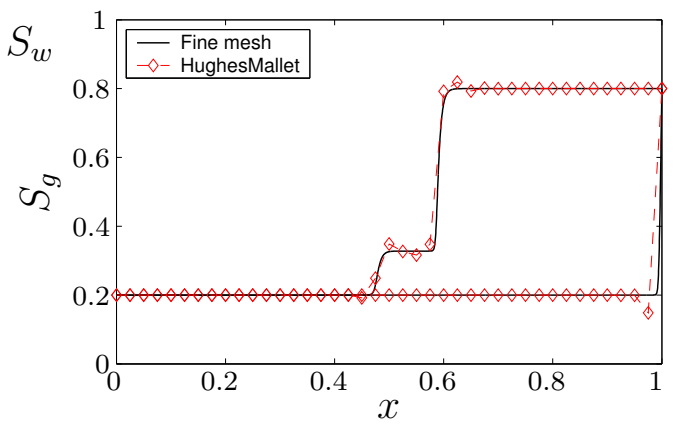

(b) Gas saturation

Figure 11. Saturation profiles of the ASGS solution ( $\boldsymbol{\tau}$ formulation given by Hughes and Mallet [29]) to the oil filtration problem on the coarse mesh.

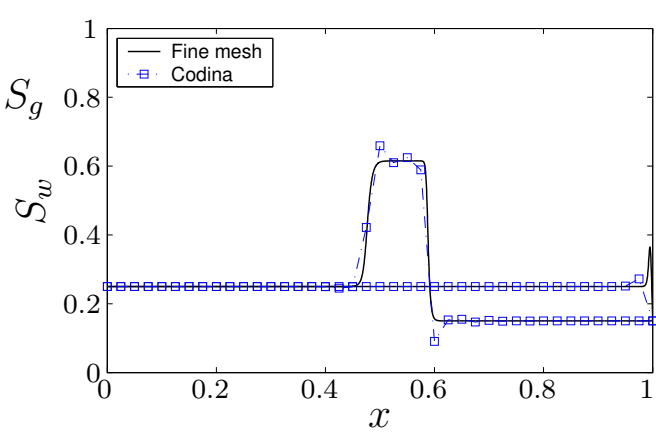

(a) Water saturation

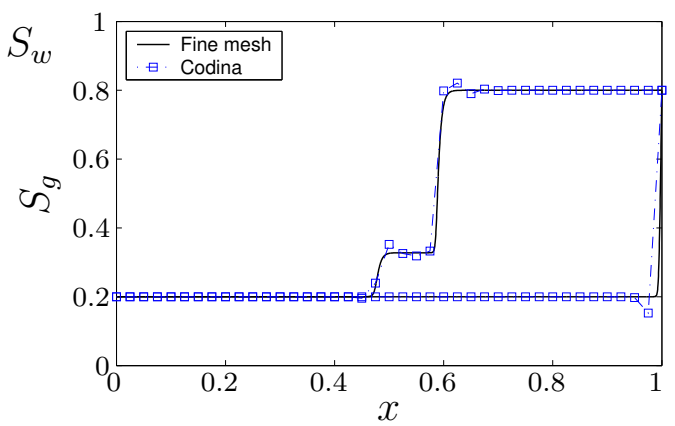

(b) Gas saturation

Figure 12. Saturation profiles of the ASGS solution ( $\boldsymbol{\tau}$ formulation given by Codina [11]) to the oil filtration problem on the coarse mesh.

different expressions of the discontinuity-capturing diffusion applied to the same ASGS method. In this case, we choose the solution obtained with the $\boldsymbol{\tau}$ matrix of Hughes and Mallet [29] - Figure 11-.

In Figure 13 we plot the results for the "canonical form" of the shockcapturing diffusion -Equation (117) - . It is found that this formulation is effective at eliminating the oscillatory behavior (compare with Figure 11), but at the cost of being a bit too diffusive.

The "quadratic form" of the discontinuity-capturing diffusion, given by Equation (118), yields the results shown in Figure 14. A single, small overshoot remains in the numerical solution: (1) at the downstream end of the 


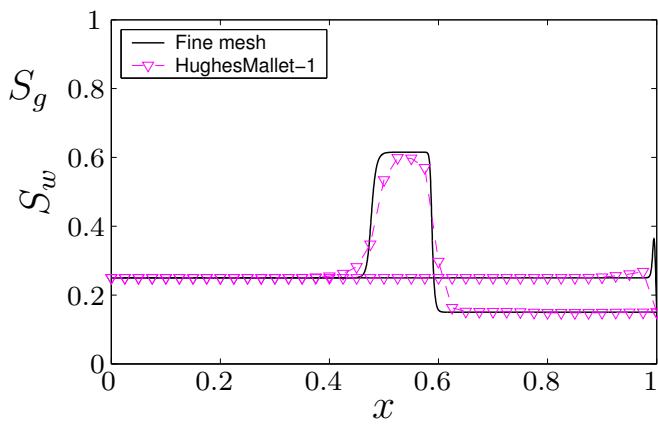

(a) Water saturation

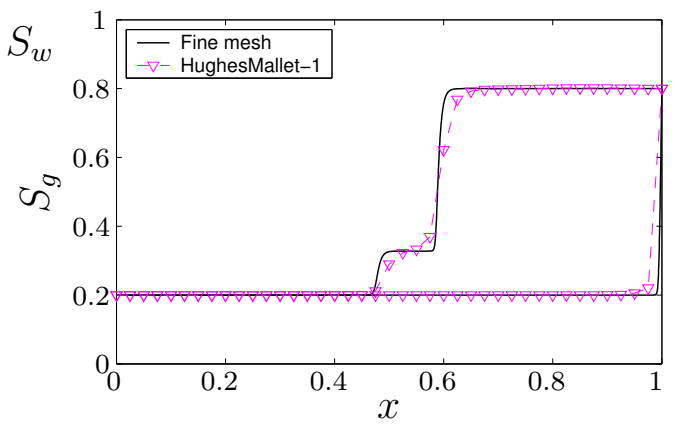

(b) Gas saturation

Figure 13. Saturation profiles of the ASGS solution to the oil filtration problem. Formulation of $\boldsymbol{\tau}$ given by [29]. Shock-capturing diffusion in "canonical form" - Equation (117) -

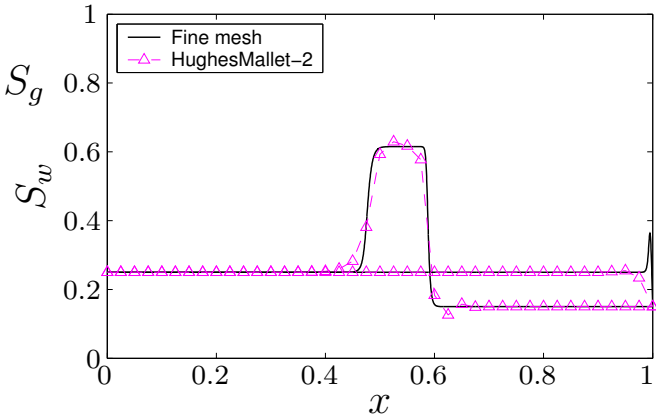

(a) Water saturation

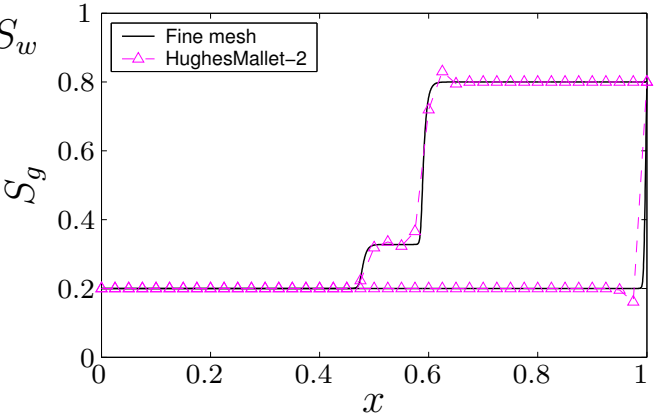

(b) Gas saturation

Figure 14. Saturation profiles of the ASGS solution to the oil filtration problem. Formulation of $\boldsymbol{\tau}$ given by [29]. Shock-capturing diffusion in "quadratic form" —Equation (118) - .

fast shock during the transient phase; and (2) at the lip of the boundary layer for stationary conditions. The solution is significantly less diffusive than that of Figure 13.

In Figure 15 we plot the numerical solution obtained when the novel "global-gradient form" of the discontinuity-capturing diffusion is employed -Equation (123) - , with the following values of the parameters:

$$
\boldsymbol{U}_{s c}=(0.5,0.5), \quad C_{s c}=2 .
$$

The method is able to remove the localized oscillatory behavior of the ASGS so- 


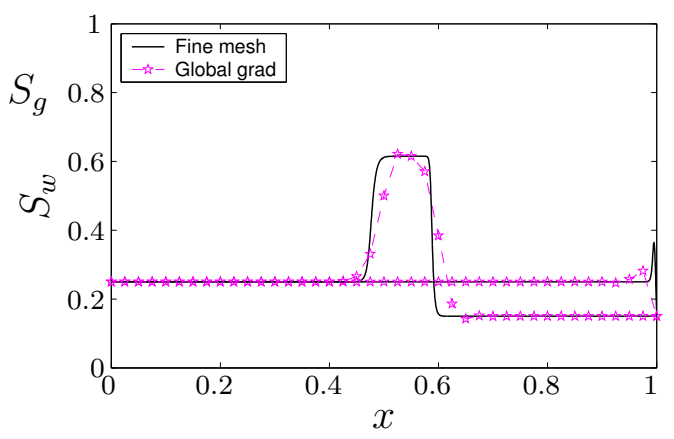

(a) Water saturation

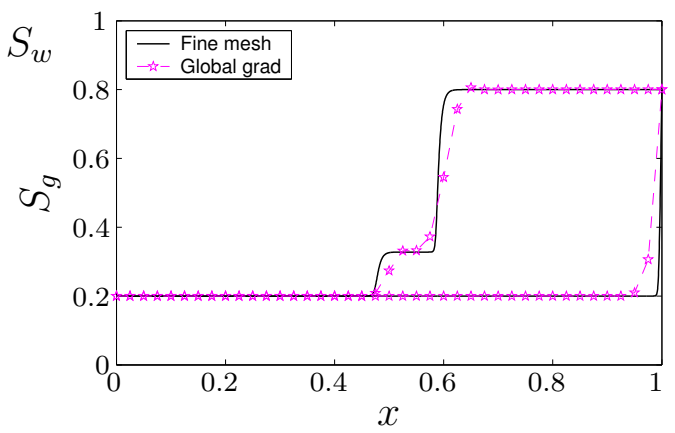

(b) Gas saturation

Figure 15. Saturation profiles of the ASGS solution to the oil filtration problem. Formulation of $\boldsymbol{\tau}$ given by [29]. Proposed formulation of shock-capturing diffusion - Equation (123) -

lution but is, for the parameters used, slightly too diffusive.

The reason for considering the novel expression of the shock-capturing diffusion as a viable alternative to existing formulations stems from the behavior of the numerical diffusion that is actually added by each method. In Figures 16, 17, and 18 we plot the profile of additional diffusion introduced by the "canonical form", the "quadratic form", and the "global-gradient form", respectively, at two different simulation times. The key observation is that, while the existing formulations add a significant amount of diffusion almost everywhere, the proposed formulation automatically introduces numerical dissipation only in the neighborhood of the sharp features of the solution. The latter is precisely the desired behavior of a discontinuity-capturing mechanism. Direct comparison of Figures 16 and 17 also explains, at a glance, why the "linear form" is significantly more diffusive than the "quadratic form".

\subsection{Water-gas injection in a reservoir}

\subsubsection{Description of the problem}

This second application involves simultaneous injection of water and gas into a porous medium that is initially filled with oil and gas (and a small amount of water), as shown in Figure 19. Initially, the medium has constant normalized saturations: $S_{w}=0.05, S_{g}=0.4$, and $S_{o}=0.55$. Gas and water are injected in such proportion that the normalized water and gas saturations at the 


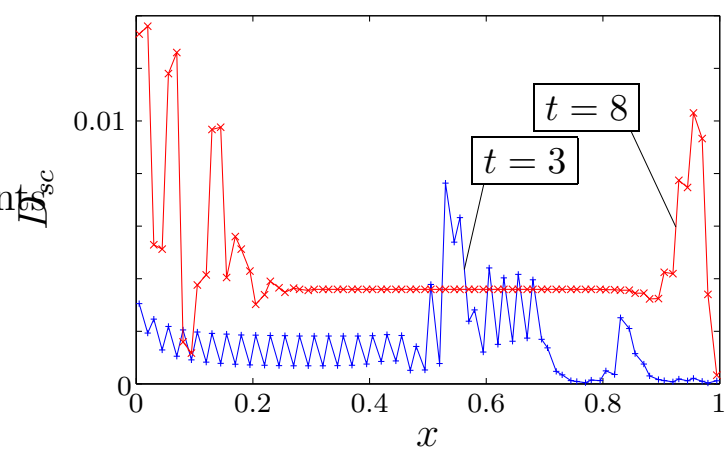

Figure 16. Profiles of shock capturing diffusion introduced by the "canonical form" - Equation (117) — at two different times.

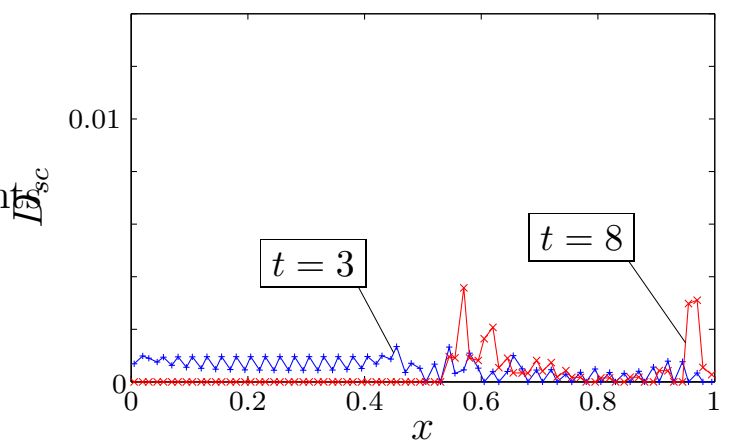

Figure 17. Profiles of shock capturing diffusion introduced by the "quadratic form" —Equation (118) — at two different times.

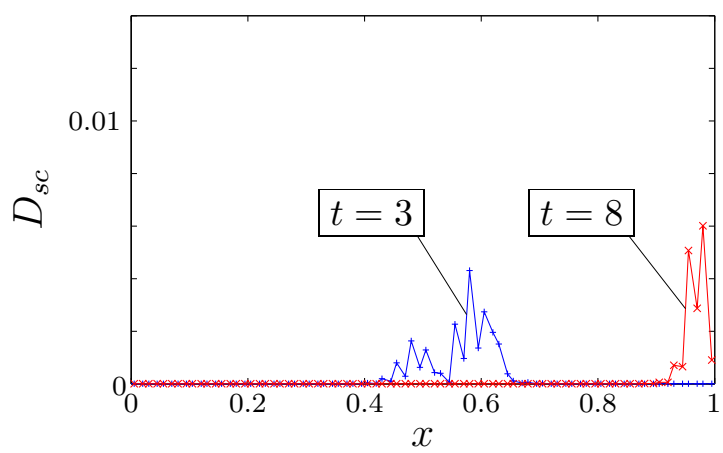

Figure 18. Profiles of shock capturing diffusion introduced by the proposed formulation -Equation (123) — at two different times. 
Injected saturations

Initial saturations

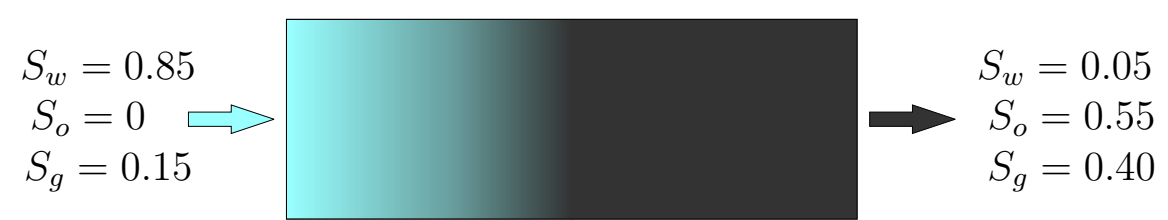

Figure 19. Sketch of the water-gas injection problem. Water and gas are injected into a medium initially filled with oil and gas.

inlet are $S_{w}=0.85$ and $S_{g}=0.15$, respectively. The injected saturations are assumed to be constant throughout the experiment. The values of initial and injected saturations used in this example are representative of a linear water-alternating-gas (WAG) injection process in a hydrocarbon reservoir after primary production $[8,51]$.

\subsubsection{Analytical solution}

The analytical solution to the water-gas injection problem described above is of type $\mathcal{R}_{1} \mathcal{S}_{1} \mathcal{S}_{2}$, that is, the 1 -wave is a rarefaction-shock and the 2 -wave is a single shock. Schematically, we write the solution structure as

$$
\boldsymbol{u}_{l} \stackrel{\mathcal{R}_{1}}{\longrightarrow} \boldsymbol{u}_{1}^{*} \stackrel{\mathcal{S}_{1}}{\longrightarrow} \boldsymbol{u}_{m} \stackrel{\mathcal{S}_{2}}{\longrightarrow} \boldsymbol{u}_{r}
$$

where $\boldsymbol{u}_{l}, \boldsymbol{u}_{m}$ and $\boldsymbol{u}_{r}$ have the same meaning as in the previous example, and $\boldsymbol{u}_{1}^{*}$ is the saturation state at which the 1-rarefaction and the 1-shock are joined - also known as the post-shock value-. The variables that need to be determined to fully characterize the solution are: the intermediate constant state $\boldsymbol{u}_{m}$, the shock speeds $\sigma_{1}$ and $\sigma_{2}$, and the post-shock state $\boldsymbol{u}_{1}^{*}$. In our particular case,

$$
\boldsymbol{u}_{1}^{*}=(0.478,0.083), \boldsymbol{u}_{m}=(0.052,0.085), \sigma_{1}=0.712, \sigma_{2}=1.280 .
$$

In Figure 20 we plot the analytical solution in saturation space. The solid line is the rarefaction curve - where the solution is continuous-, and the dashed lines are the shock curves, which correspond to discontinuities.

The fluid saturation profiles of the analytical solution to the water-gas injection problem are shown in Figure 21. Because the capillarity-free solution is self-similar, the profiles are plotted against the similarity variable $\zeta=x / t$. The most relevant features of the solution are: 


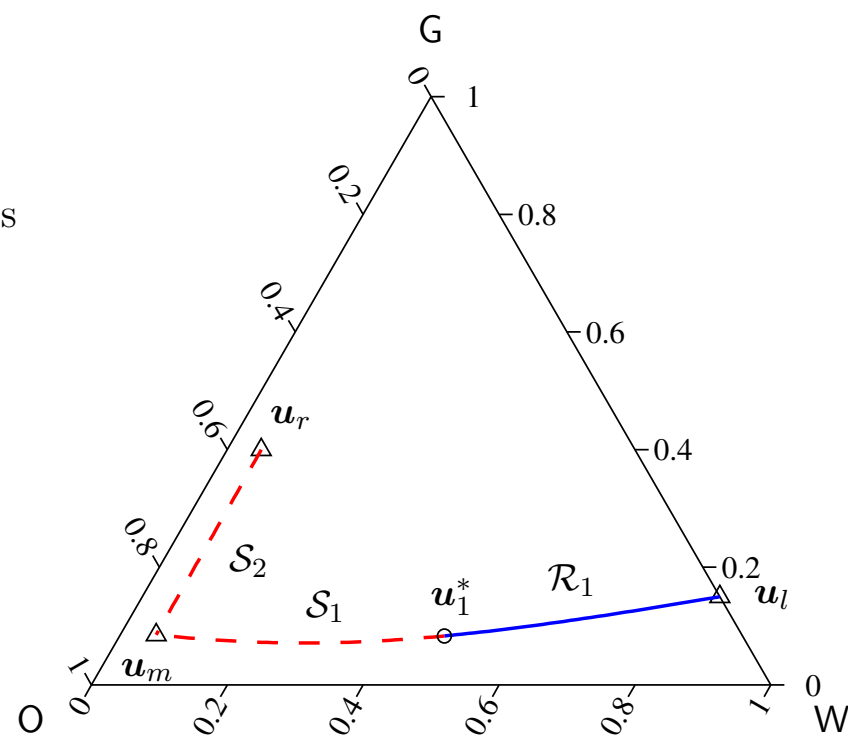

Figure 20. Saturation path of the exact solution to the water-gas injection problem. The 1 -wave is a rarefaction-shock and the 2 -wave is a shock $\left(\mathcal{R}_{1} \mathcal{S}_{1} \mathcal{S}_{2}\right.$ solution).

1. Oil and gas are produced by a sequence of two waves. The slow wave involves mainly displacement of oil by injected water, and the fast wave corresponds to a displacement of gas by oil.

2. An oil bank - intermediate state with higher oil saturations than those of the initial and injected states - is formed. This is characteristic of water flood processes in the presence of free gas $[46,65]$.

\subsubsection{Comparison of numerical solutions}

We compute a "reference" numerical solution to the water-gas injection problem with small capillary diffusion coefficients:

$$
\epsilon_{w}=0.001, \quad \epsilon_{g}=0.002 .
$$

We use the standard Galerkin formulation on a very fine mesh of 4000 elements $\left(h=2.5 \times 10^{-4}\right)$, and a Crank-Nicolson time integration scheme 


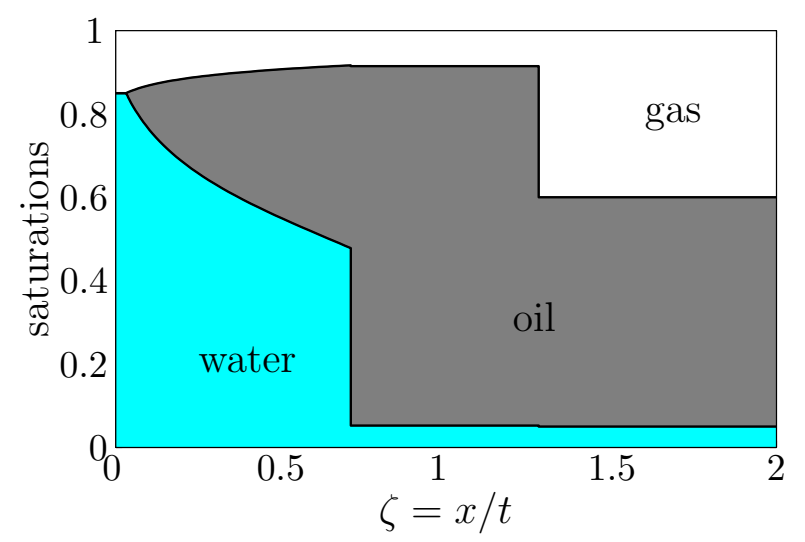

Figure 21. Saturation profiles of the exact solution to the water-gas injection problem. Saturations of each phase are plotted against the similarity variable $\zeta=x / t$.

with $\delta t=5 \times 10^{-5}$. For this space and time discretization, the element Peclet and Courant numbers are, respectively:

$$
\begin{aligned}
& P e:=\frac{\sigma_{\max } h}{\epsilon_{\min }} \approx 0.3, \\
& C o:=\frac{\sigma_{\max } \delta t}{h} \approx 0.25 .
\end{aligned}
$$

In Figure 22 we plot the water and gas saturation profiles of the "reference" numerical solution at $t=0.5$, together with the capillarity-free analytical solution. The numerical solution correctly captures the location and magnitude of the shocks, and provides an accurate representation of the rarefaction fan. As was the case in the oil filtration example, additional simulations with different space and time discretizations, and different capillary diffusion coefficients, confirm convergence of the standard Galerkin method to the entropy solution of the problem.

The saturation path of the reference numerical solution is shown in Figure 23. In this case, the saturation path agrees very well with that of the capillarity-free solution, not only along the rarefaction, but also along shocks. The close matching along shock curves is not particularly relevant. It has to do with the fact that the shocks join states with the similar water or gas saturation, and that an isotropic capillary diffusion tensor is used. 


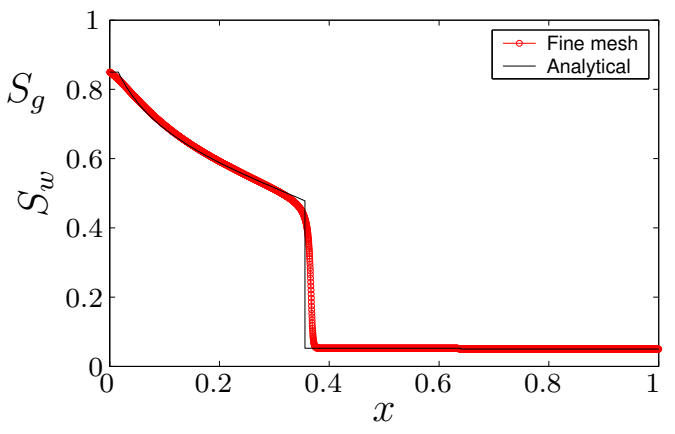

(a) Water saturation

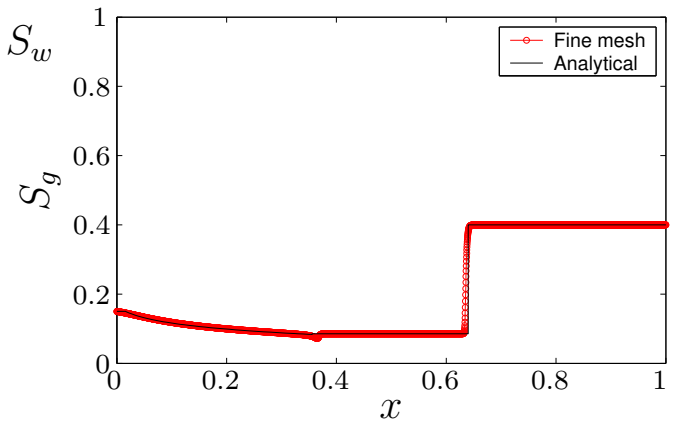

(b) Gas saturation

Figure 22. Saturation profiles of the standard Galerkin solution to the water-gas injection problem on a fine mesh of 4000 elements, and comparison to the analytical solution of the capillarity-free case. Results are shown at time $t=0.5$.

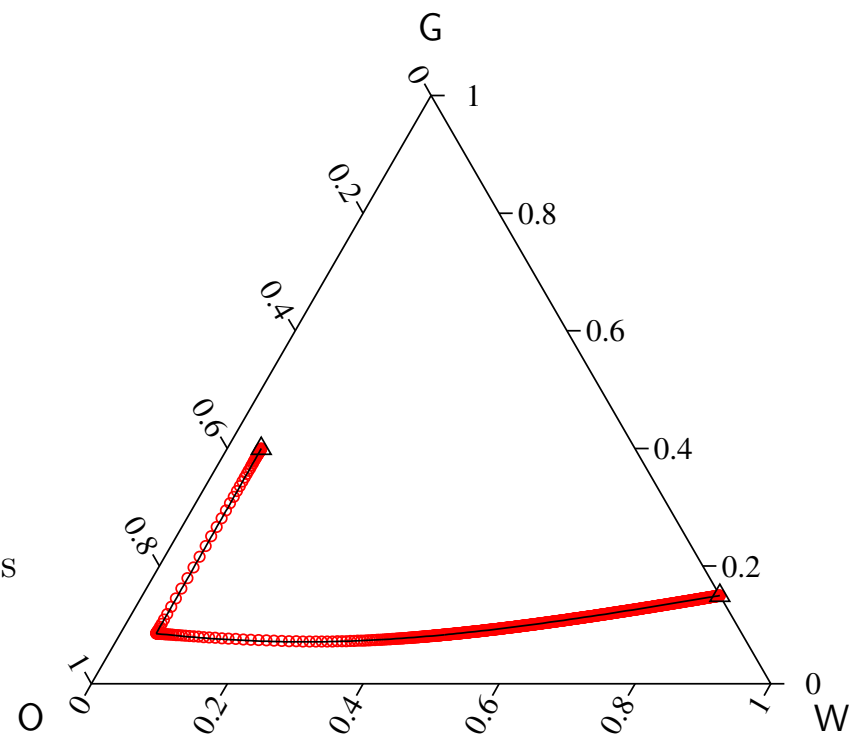

Figure 23. Comparison of the saturation path obtained by the standard Galerkin method on a fine mesh of 4000 elements (dotted curve) and the exact solution (solid curve) of the water-gas injection problem. 


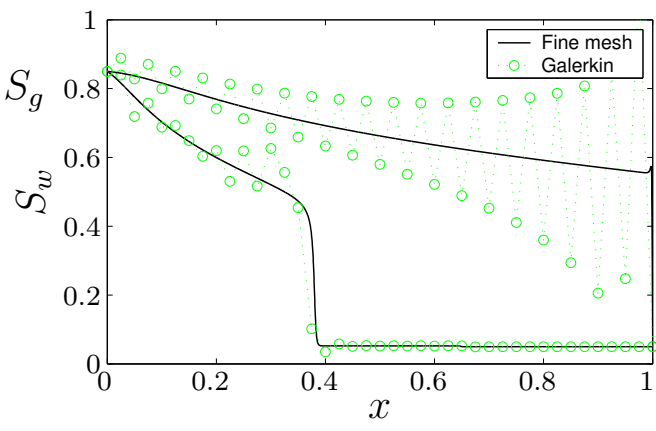

(a) Water saturation

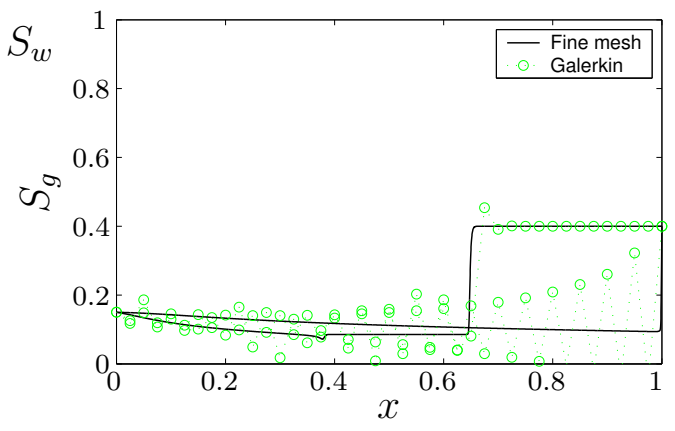

(b) Gas saturation

Figure 24. Saturation profiles of the standard Galerkin solution to the water-gas injection problem on a coarse mesh of 40 elements. Results are shown at times $t=0.5$ and $t=2$.

Standard Galerkin solutions. The water-gas injection problem is solved with the same physical parameters on a much coarser mesh of 40 elements and a time step $\delta t=0.005$. The element Peclet number is now $P e \approx 30$, and the element Courant number remains $C o \approx 0.25$. The results are shown in Figure 24 at two different simulation times $(t=0.5$ and $t=2)$, and compared with the reference numerical solution. Clearly, the standard Galerkin solution on the coarse mesh is unstable. The Galerkin solution is completely oscillatory, especially after the process reaches a quasi-steady state.

Algebraic subgrid scale solutions. The numerical solution produced by the ASGS method with the $\boldsymbol{\tau}$ formulation of Hughes and Mallet [29] is shown in Figure 25. The behavior of the method is remarkable, considering that a very coarse mesh of only 40 elements was used. The stabilizing term is able to remove the global oscillatory behavior of the standard Galerkin method. The solution is also extremely accurate and preserves a sharp definition of the shocks and boundary layers.

It should be noted, however, that other formulations of the matrix $\boldsymbol{\tau}$ of stabilizing coefficients — such as those proposed in [11,59] — do not yield the impressive results of Figure 25. In some cases they even fail to converge, emphasizing the importance of an appropriate choice of $\boldsymbol{\tau}$ for each particular problem. 


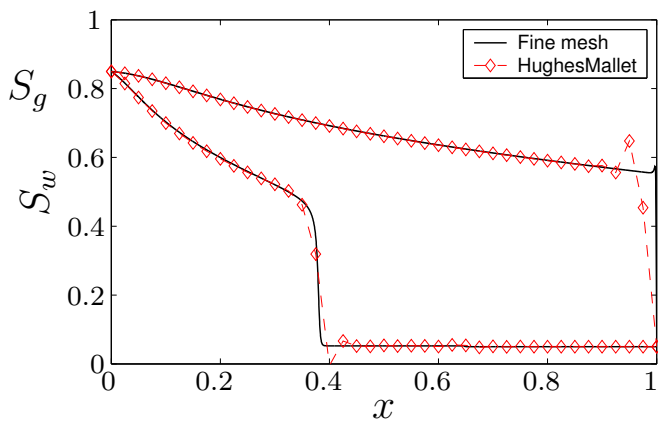

(a) Water saturation

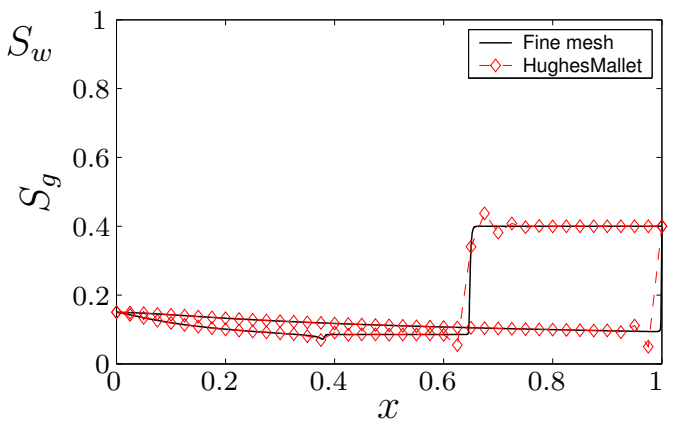

(b) Gas saturation

Figure 25. Saturation profiles of the ASGS solution ( $\boldsymbol{\tau}$ formulation given by Hughes and Mallet [29]) to the water-gas injection problem on the coarse mesh.

Stabilized solutions with shock capturing diffusion. Despite the effective stabilization of the ASGS method with the matrix of stabilizing coefficients given by Hughes and Mallet [29], some local overshooting is still present in the solution (see Figure 25). We make use of a discontinuitycapturing technique to remove the spurious wiggles. In Figure 26 we plot the numerical solution obtained after using the ASGS method above in conjunction with the proposed "global-gradient form" of the shock-capturing diffusion -Equation (123) — with the following parameters:

$$
\boldsymbol{U}_{s c}=(0.5,0.5), \quad C_{s c}=2 .
$$

The computed solution retains exceptional accuracy in the smooth regions - the rarefaction fan and the constant saturation states - while effectively enhancing stability near the sharp gradients.

The profile of additional diffusion introduced by the discontinuity-capturing term is plotted - at simulation times $t=0.5$ and $t=2$ - in Figure 27. It is apparent that the amount of artificial diffusion is negligible everywhere, except: (1) in the vicinity of both shocks during transient conditions; (2) near the boundary layer for quasi-steady conditions. All other formulations of shock-capturing diffusion described in Section 3.6 are either less effective or even fail to converge. 


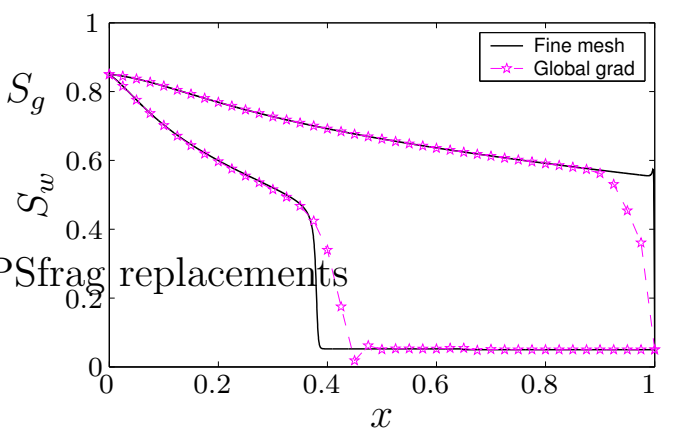

(a) Water saturation

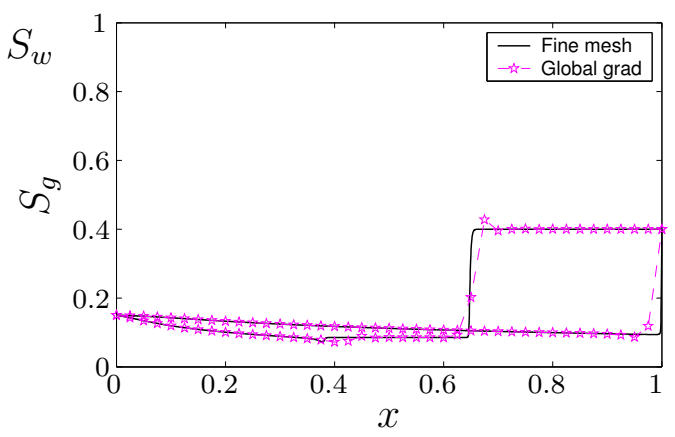

(b) Gas saturation

Figure 26. Saturation profiles of the ASGS solution to the water-gas injection problem. Formulation of $\boldsymbol{\tau}$ given by [29]. Proposed formulation of shock-capturing diffusion - Equation (123) -

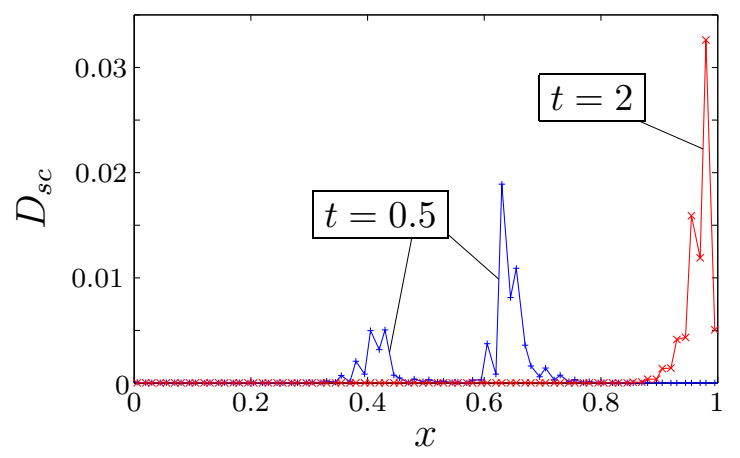

Figure 27. Profiles of shock capturing diffusion introduced by the proposed formulation -Equation (123) - at two different times.

\section{Conclusions}

We have presented a fairly general formulation for the numerical solution of nonlinear systems of conservation laws, and applied it to the equations of one-dimensional three-phase flow through porous media. The method is based on the original framework presented in [26], and entails a multiplescale decomposition of the solution into resolved and unresolved scales. It is precisely the effect of the unresolved - subgrid - scales on the resolved - grid - scales that introduces a stabilizing term in the formulation. Key distinctive features of the formulation developed herein are: (1) the multiscale 
split is performed before any linearization of the equations (which are kept in conservation form); (2) the multiscale solution is not reconstructed from point values of coarse-scale and subgrid-scale solutions; and (3) a novel shockcapturing technique is proposed to further enhance stability of the solution in the neighborhood of strong gradients.

From the results presented in Section 4, we conclude that the proposed stabilized method yields numerical solutions of exceptional quality to challenging, highly nonlinear, nearly hyperbolic problems. Solutions computed on very coarse grids display excellent stability and accuracy. The algebraic subgrid model employed is quite sensitive, however, to the choice of the matrix of stabilizing coefficients $\boldsymbol{\tau}$. The definition of $\boldsymbol{\tau}$ given by Hughes and Mallet [29], which requires the solution of an eigenvalue problem, seems to be the most applicable to the type of problems considered in this paper. This observation is further confirmed by numerical experiments with other onedimensional systems that become strictly hyperbolic in the limit of vanishing diffusion - such as the shallow-water equations and the Euler equations of gas dynamics - Application of the methodology to these interesting problems will be reported in subsequent publications.

The novel formulation of the discontinuity-capturing diffusion — coined "global-gradient form" - provides an alternative to existing formulations. The simulations of Section 4 clearly show that, in contrast to the canonical expressions, the numerical diffusion introduced by the proposed formulation is confined to the vicinity of discontinuities in the solution.

Several issues deserve further investigation. One of the topics that is currently being addressed is the study of a different approximation to the subscales. In particular, we are interested in a numerical approximation of the subgrid scale problem with appropriate basis functions - high-order finite elements, wavelets, etc.-, which are potentially capable of capturing the sharp features of the solution that the coarse mesh is unable to resolve.

\section{ACKNOWLEDGEMENTS}

This work was supported in part by the U.S. Department of Energy under Contract No. DE-AC03-76SF00098. Funding provided by Barrié de la Maza, Jane Lewis, and Repsol-YPF fellowships, awarded to the first author, is also gratefully acknowledged. 


\section{References}

[1] T. Arbogast. Numerical subgrid upscaling of two-phase flow in porous media. In Z. Chen, R. E. Ewing, and Z.-C. Shi, editors, Numerical Treatment of Multiphase Flow in Porous Media, volume 552 of Lecture Notes in Physics, pages 35-49, Berlin, 2000. Springer.

[2] T. Arbogast. Implementation of a locally conservative numerical subgrid upscaling scheme for two-phase Darcy flow. Comput. Geosci., 6(3-4):453481, 2002.

[3] K. Aziz and A. Settari. Petroleum Reservoir Simulation. Elsevier, London, 1979.

[4] C. Baiocchi, F. Brezzi, and L. P. Franca. Virtual bubbles and Galerkin/leastsquares type methods (Ga.L.S). Comput. Methods Appl. Mech. Engrg., 105:125-141, 1993.

[5] F. Brezzi, L. P. Franca, T. J. R. Hughes, and A. Russo. $b=\int g$. Comput. Methods Appl. Mech. Engrg., 145:329-339, 1997.

[6] A. N. Brooks and T. J. R. Hughes. Streamline upwind Petrov-Galerkin formulations for convection dominated flows with particular emphasis on the incompressible Navier-Stokes equations. Comput. Methods Appl. Mech. Engrg., 32:199-259, 1982.

[7] G. Chavent and J. Jaffré. Mathematical Models and Finite Elements for Reservoir Simulation, volume 17 of Studies in Mathematics and its Applications. Elsevier, North-Holland, 1986.

[8] J. R. Christensen, E. H. Stenby, and A. Skauge. Review of WAG field experience. SPERE, 4(2):97-106, April 2001.

[9] R. Codina. A discontinuity-capturing crosswind-dissipation for the finite element solution of the convection-diffusion equation. Comput. Methods Appl. Mech. Engrg., 110:325-342, 1993.

[10] R. Codina. Comparison of some finite element methods for solving the diffusion-convection-reaction equation. Comput. Methods Appl. Mech. Engrg., 156:185-210, 1998.

[11] R. Codina. On stabilized finite element methods for linear systems of convection-diffusion-reaction equations. Comput. Methods Appl. Mech. Engrg., 188:61-82, 2000. 
[12] R. Codina. A stabilized finite element method for generalized stationary incompressible flows. Comput. Methods Appl. Mech. Engrg., 190:2681-2706, 2001.

[13] R. Codina. Stabilized finite element approximation of transient incompressible flows using orthogonal subscales. Comput. Methods Appl. Mech. Engrg., 191(39-40):4295-4321, 2002.

[14] R. Codina and J. Blasco. Analysis of a stabilized finite element approximation of the transient convection-diffusion-reaction equation using orthogonal subscales. Comput. Visual. Sci., 4(3):167-174, 2002.

[15] E. G. Dutra do Carmo and A. C. Galeão. Feedback Petrov-Galerkin methods for convection-dominated problems. Comput. Methods Appl. Mech. Engrg., 88:1-16, 1991.

[16] L. P. Franca, A. Nesliturk, and M. Stynes. On the stability of residual free bubbles for convection-diffusion problems and their approximation by a twolevel finite element method. Comput. Methods Appl. Mech. Engrg., 166:35-49, 1998.

[17] L. P. Franca (editor). Special issue on "Advances in stabilized methods in computational mechanics". Comput. Methods Appl. Mech. Engrg., 166(1-2), 1998.

[18] A. C. Galeão and E. G. Dutra do Carmo. A consistent approximate upwind Petrov-Galerkin method for convection-dominated problems. Comput. Methods Appl. Mech. Engrg., 68:83-95, 1988.

[19] K. Garikipati and T. J. R. Hughes. A study of strain localization in a multiple scale framework - The one-dimensional problem. Comput. Methods Appl. Mech. Engrg., 159:193-222, 1998.

[20] K. Garikipati and T. J. R. Hughes. A variational multiscale approach to strain localization -Formulation for multidimensional problems. Comput. Methods Appl. Mech. Engrg., 188:39-60, 2000.

[21] R. E. Guzmán. Mathematics of Three-Phase Flow. PhD Dissertation, Stanford University, July 1995.

[22] S. M. Hassanizadeh and W. G. Gray. Toward an improved description of the physics of two-phase flow. Adv. Water Resour., 16(1):53-67, 1993. 
[23] G. Hauke. A simple subgrid scale stabilized method for the advectiondiffusion-reaction equation. Comput. Methods Appl. Mech. Engrg., 191:2925$2947,2002$.

[24] G. Hauke and A. García-Olivares. Variational subgrid scale formulations for the advection-diffusion-reaction equation. Comput. Methods Appl. Mech. Engrg., 190:6847-6865, 2001.

[25] T. Y. Hou and X.-H. Wu. A multiscale finite element method for elliptic problems in composite materials and porous media. J. Comput. Phys., 134:169$189,1997$.

[26] T. J. R. Hughes. Multiscale phenomena: Green's functions, the Dirichletto-Neumann formulation, subgrid scale models, bubbles and the origins of stabilized methods. Comput. Methods Appl. Mech. Engrg., 127:387-401, 1995.

[27] T. J. R. Hughes, G. R. Feijóo, L. Mazzei, and J.-B. Quincy. The variational multiscale method - a paradigm for computational mechanics. Comput. Methods Appl. Mech. Engrg., 166:3-24, 1998.

[28] T. J. R. Hughes, L. P. Franca, and G. M. Hulbert. A new finite element formulation for computational fluid dynamics: VIII. The Galerkin least-squares method for advective-diffusive equations. Comput. Methods Appl. Mech. Engrg., 73:173-189, 1989.

[29] T. J. R. Hughes and M. Mallet. A new finite element formulation for computational fluid dynamics: III. The generalized streamline operator for multidimensional advective-diffusive systems. Comput. Methods Appl. Mech. Engrg., 58:305-328, 1986.

[30] T. J. R. Hughes and M. Mallet. A new finite element formulation for computational fluid dynamics: IV. A discontinuity-capturing operator for multidimensional advective-diffusive systems. Comput. Methods Appl. Mech. Engrg., 58:329-336, 1986.

[31] T. J. R. Hughes, M. Mallet, and A. Mizukami. A new finite element formulation for computational fluid dynamics: II. Beyond SUPG. Comput. Methods Appl. Mech. Engrg., 54:341-355, 1986.

[32] T. J. R. Hughes, L. Mazzei, and K. E. Jansen. Large Eddy Simulation and the variational multiscale method. Comput. Visual. Sci., 3:47-59, 2000.

[33] T. J. R. Hughes, L. Mazzei, and A. A. Oberai. The multiscale formulation of large eddy simulation: Decay of homogeneous isotropic turbulence. Phys. Fluids, 13(2):505-512, 2001. 
[34] T. J. R. Hughes, A. A. Oberai, and L. Mazzei. Large eddy simulation of turbulent channel flows by the variational multiscale method. Phys. Fluids, 13(6):1784-1799, 2001.

[35] K. E. Jansen, S. S. Collis, C. Whiting, and F. Shakib. A better consistency for low-order stabilized finite element methods. Comput. Methods Appl. Mech. Engrg., 174:153-170, 1999.

[36] C. Johnson. Numerical Solution of Partial Differential Equations by the Finite Element Method. Cambridge University Press, New York, 1987.

[37] C. Johnson. A new approach to algorithms for convection problems which are based on exact transport+projection. Comput. Methods Appl. Mech. Engrg., 100:45-62, 1992.

[38] C. Johnson and A. Szepessy. On the convergence of a finite element method for a nonlinear hyperbolic conservation law. Math. Comp., 49(180):427-444, 1987.

[39] C. Johnson, A. Szepessy, and P. Hansbo. On the convergence of shockcapturing streamline diffusion finite element methods for hyperbolic conservation laws. Math. Comp., 54(189):107-129, 1990.

[40] R. Juanes and T. W. Patzek. Analytical solution to the Riemann problem of three-phase flow in porous media. Transp. Porous Media, 2002. (Submitted).

[41] R. Juanes and T. W. Patzek. Multiple scale stabilized finite elements for the simulation of tracer injections and waterflood. In SPE/DOE Thirteenth Symposium on Improved Oil Recovery, Tulsa, OK, April 13-17, 2002. (SPE 75231).

[42] R. Juanes and T. W. Patzek. Multiscale finite element methods for miscible and immiscible flow in porous media. J. Hydraul. Res., 2002. (Accepted).

[43] R. Juanes and T. W. Patzek. Relative permeabilities for strictly hyperbolic models of three-phase flow in porous media. Transp. Porous Media, 2002. (Submitted).

[44] R. Juanes and T. W. Patzek. Strictly hyperbolic models of co-current threephase flow with gravity. Transp. Porous Media, 2002. (Submitted).

[45] R. Juanes and T. W. Patzek. Three-phase displacement theory: Towards an improved description of relative permeabilities. In SPE Annual Technical Conference and Exhibition, San Antonio, TX, September 29-October 2, 2002. (SPE 77539). 
[46] J. R. Kyte, R. J. Stanclift Jr., S. C. Stephan Jr., and L. A. Rapoport. Mechanism of water flooding in the presence of free gas. Petrol. Trans. AIME, 207:215-221, 1956.

[47] L. W. Lake. Enhanced Oil Recovery. Prentice-Hall, Englewood Cliffs, NJ, 1989.

[48] R. Lenormand. Pattern growth and fluid displacements through porous media. Physica A, 140:114-123, 1986.

[49] R. J. LeVeque. Numerical Methods for Conservation Laws. Birkhäuser Verlag, Berlin, second edition, 1992.

[50] M. C. Leverett and W. B. Lewis. Steady flow of gas-oil-water mixtures through unconsolidated sands. Petrol. Trans. AIME, 142:107-116, 1941.

[51] D. Marchesin and B. J. Plohr. Wave structure in WAG recovery. SPEJ, 6(2):209-219, June 2001.

[52] J. E. Marsden and T. J. R. Hughes. Mathematical Foundations of Elasticity. Prentice-Hall, Englewood Cliffs, NJ, 1983. Reprinted with corrections, Dover, New York, 2000.

[53] A. Masud and T. J. R. Hughes. A stabilized mixed finite element method for Darcy flow. Comput. Methods Appl. Mech. Engrg., 191(39-40):4341-4370, 2002.

[54] M. Muskat. Physical Principles of Oil Production. McGraw-Hill, New York, 1949.

[55] A. A. Oberai and P. M. Pinsky. A multiscale finite element method for the Helmholtz equation. Comput. Methods Appl. Mech. Engrg., 154:281-297, 1998.

[56] A. A. Oberai and P. M. Pinsky. A residual-based finite element method for the Helmholtz equation. Int. J. Numer. Meth. Engrg., 49(3):399-419, 2000.

[57] D. W. Peaceman. Fundamentals of Numerical Reservoir Simulation, volume 6 of Developments in Petroleum Science. Elsevier, Amsterdam, 1977.

[58] M. Peszynska, M. F. Wheeler, and I. Yotov. Mortar upscaling for multiphase flow in porous media. Comput. Geosci., 6(1):73-100, 2002.

[59] F. Shakib, T. J. R. Hughes, and Z. Johan. A new finite element formulation for computational fluid dynamics: X. The compressible Euler and Navier-Stokes equations. Comput. Methods Appl. Mech. Engrg., 89:141-219, 1991. 
[60] H. L. Stone. Probability model for estimating three-phase relative permeability. JPT, 23(2):214-218, February 1970. Petrol. Trans. AIME, 249.

[61] H. L. Stone. Estimation of three-phase relative permeability and residual oil data. J. Can. Petrol. Technol., 12(4):53-61, 1973.

[62] T. E. Tezduyar and Y. J. Park. Discontinuity-capturing finite element formulations for nonlinear convection-diffusion-reaction equations. Comput. Methods Appl. Mech. Engrg., 59:307-325, 1986.

[63] V. Thomée. Galerkin Finite Element Methods for Parabolic Problems, volume 25 of Springer Series in Computational Mathematics. Springer-Verlag, Berlin, 1997.

[64] M. R. Todd, P. M. O'Dell, and G. J. Hirasaki. Methods of increased accuracy in numerical reservoir simulators. SPEJ, 12(6):515-530, December 1972. Petrol. Trans. AIME, 253.

[65] G. P. Willhite. Waterflooding, volume 3 of SPE Textbook Series. Society of Petroleum Engineers, Richardson, TX, 1986.

[66] E. Zauderer. Partial Differential Equations of Applied Mathematics. Series in Pure and Applied Mathematics. John Wiley \& Sons, New York, 1983. 